\title{
MATERIALIEN
}

Anton Bierl and William M. Calder III

\section{FRIEDRICH NIETZSCHE: “ABRISS DER GESCHICHTE DER BEREDSAMKEIT" \\ A NEW EDITION}

For Albert Henrichs

\section{I.}

\section{Introduction}

If the sands of Egypt should yield the barest scraps of Plato's lecture notes, publication would be assured and a vast secondary bibliography would soon follow. A case certainly could be made - whether cogent or not is not at issue here - that Friedrich Nietzsche is romparable. Scraps of his lecture notes also deserve publication. The most modest addition to the corpus of so great and influential a thinker may allow posterity better to understand him. That is what three Germanists, Nietzsche specialists, thought. The Director of the Goethe-Schiller Archive in Weimar and the Oxford University Press agreed with them. And so we have:

Friedrich Nietzsche on Rhetoric and Language, Edited and Translated with a Critical Introduction by Sander L. Gilman, Carole Blair, David J. Parent, New York/Oxford, 1989 (henceforth cited: GBP).

The new text in the volume that is of greatest interest to historians of Greek literature and its reception is the brief "Anhang: Abriss der Geschichte der Beredsamkeit". The essay is entirely historical. It is written in the midst of the controversy on Geburt that pitted Nietzsche against the greatest Hellenist of modern times ${ }^{1}$. Wintersemester $1872 / 73$ was the most discouraging

' See William M. Calder III, "The Wilamowitz-Nietzsche struggle: New documents and a reappraisal". Nietzsche-Studien 12 (1983), 214-254 and Jaap Mansfeld, "The WilamowitzNietzsche struggle: Another new document and some further comments", Nietzsche-Studien 15 (1986), $41-58$. 
in Nietzsche's brief professional career. All the students in his institute boycotted him. He writes Erwin Rohde in November 1872²:

Hier ist das nächste, mich etwas bedrückende Faktum, daß an unserer Universität die Philologen a usgeblieben sind, für dies Wintersemester: ein ganz einziges Phänomen, das Du Dir wohl eben so deuten wirst wie ich. [...] Mit äußerster Noth habe ich ein Colleg über Rhetorik d $\langle$ er $\rangle$ $\mathrm{G}\langle$ riechen〉 und $\mathrm{R}\langle$ ömer $\rangle$ zu Stande gebracht, mit 2 Zuhörern dh. einem Germanisten und einem Juristen.

Johannes Stroux adds ${ }^{3}$ :

Mitteilungen etc. "Die andern angekündigten Vorlesungen sind nicht zu stande gekommen."

He had little reason to excel and the Abriss confirms this.

For classical admirers of Nietzsche; proximity to Geburt adds considerably to their interest in the Abriss. Geburt has exerted a lasting influence on subsequent scholarship both in Greek tragedy and the religion of Dionysos ${ }^{4}$. What does this new essay reveal of Nietzsche's competence in the history of Greek literature? Can its composition and content cast any light on Geburt? What follows is concerned with this essay solely (= GBP $166-193)$ and aims to provide an authoritative text with commentary and evaluate what it tells us about Nietzsche as a professional philologist.

Why is our article necessary? No editio princeps can be expected to be definitive. But readers of an editio princeps, that means by definition an edition that cannot be compared with any other printed edition but only with the autograph, to which few readers will have access, may reasonably expect that: 1. The word by word transcription is accurate with dubious readings noted. 2. Any changes made by the editors that diverge from the author's manuscript

${ }^{2}$ KGB II. 3, No. 272; for Rohde's reply of 14 November 1872 seee KGB II. 4, No. 379. Nietzsche proposed a fuller course of lectures on ancient rhetoric for SS 1874. Because of the lack of students they were never delivered. The earlier confusion between the two sets of lectures has been clarified. Otto Crusius had earlier published the 1874 notes as though the notes for WS 1872/73: see Nietzische's Werke XVIII. 3. II, Philologica II. Unveröffentlichtes zur Litteraturgeschichte, Rhetorik und Rhytbmik, ed. by Otto Crusius, Leipzig, 1912, 199-236. Now the tecollections of one of Nietzsche's two students in $1872 / 73$, Louis Kelterborn (1853-1910) prove that the Abriss must be dated to Novemiber 1872: see GBP, $\mathrm{x}-\mathrm{xi}$ and Sander L. Gilman, Begegnungen mit Nietasche, Bonn, ${ }^{2} 1985,111 \mathrm{f}$.

3 Johannes Stroux, Nietzsches Professur in. Basel, Jena, 1925, 97. This book remains authoritative for its subject and is unknown to GBP.

4 See Martin Vogel, Apollinisch und Dionysisch: Geschichte eines genialen Irrtums, Studien zur Musikgeschichte des 19. Jahrhunderts 6, Regensburg, 1966; Albert Henrichs, "Loss-of self, suffering, violence: The modern view of Dionysus from Nietzsche to Girard", HSCP 88 (1984), 205-240, esp. 219-234, and recently Anton Bierl, Dionysos und die griechiscbe Tragödiẹ. Politische und 'metatheatralische' Aspekte im Text, Tübingen 1991, 1-25. 
are clearly indicated so that the reader may himself judge whether they are convincing.

3. The editors are expert in the language and subject matter of the material they are editing for the first time.

4. The editors competently evaluate the new material and place it within the context of what had been known.

5. The editors, if they append a translation, are able to translate their text accurately.

In short, the first printed edition should relieve the reader from any need to consult the Weimar manuscript.

How do the editors (henceforth: GBP) fulfill these reasonable expectations? Our new edition and its commentary answer this question ${ }^{5}$. But because we shall not in our commentary draw attention to every error in the editio princeps, to justify our edition we provide illustrative examples for the five criteria above:

1. "2a. VIII 57" (GBP 166) conveys precisely nothing to a reader. Nietzsche wrote "La. VIII 57", that is he refers to Diogenes Laertius 8. 57. Diogenes was the center of early Nietzsche's scholarly work (see KG II. 1, 75-245) and three editors of Nietzsche miss a reference to him. At GBP 176 we find someone called "Dionys. Laert.". What Nietzsche wrote was "Dionys 〈ius Halicarnaseus〉 Isocr〈ates〉". GBP (186) read: "Der Academiker Corneades [sic], der Richter Diogenes, der Peripatetiker Aristolaus. Die Wirkung war so gross, dass Cato die Betreuung der Gesandten antrug." What Nietzsche wrote was "Der Academ. Carneades, der Stoiker Diogenes, der Peripat. Critolaus. Die Wirkung war so gross, dass Cato die Entfernung der Gesandten antrug." (See infra n. 214). Errors of this sort are numerous (see e.g. infra nn. $53,69,73,142,187,214,253)$. One cannot trust the transcription.

2. GBP (xxi) assert: "The text is transcribed as it stands in the manuscript." This is an untruth. They add words, e.g. "sind" at GBP 172. 6, without indication. They even transpose whole passages within Nietzsche's manuscripr without a word of warning or justification (see infra nn. 26, 30, 38, 52, 75, 154). They fill out abbreviations in Nietzsche's manuscript without any indication. At GBP 172 we have "Während des pelopponesischen Kriegs".

"The text of "Darstellung der Antiken Rhetorik" (GBP 2-166) scems better, not least because there is less Greek and Jatin in it; but there are errors of an ignorance that expands "Dionys. de compos. verbor." (GBP 47) to De Composila Verborum and gives us a new Roman rhetorician Sulpitius Victor (GBP 200 n. 10)! When Nietzsche writes that biography "ist kein griechisches Wort" (GBP 46), GBP have no idea that he is wrong (see Dam. lsid. 8). Expectedly they cannot distinguish xi from delta (GBP 16). And so on. 
If in November 1872 Nietzsche were unable to spell "peloponnesischen", that would provide revealing evidence that Wilamowitz was right to urge that Nietzsche resign his chair. In fact Nietzsche wrote "pelop" and GBP cannot spell the word. All expanded abbreviations must be indicated to avoid such confusion.

3. Do they know German? We have "Grosser Lob" (GBP 170) and at GBP 180 "flog Demosthenes nach Calauria". Presumably in a plane. Three American professors of German cannot distinguish between fliegen and flieben. They do not know Latin. We have the "tenue dia[poreticum] g[enus]"(!) (GBP 172) for "tenue dic〈endi〉g〈enus〉". They do not know the simplest Greek nor indeed the Greek alphabet. On the first page (GBP 166) they write

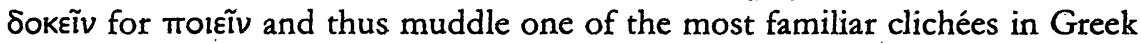
(see infra n. 19). Again and again they print unintelligible Greek.

4. The editors have no idea of the history of Greek oratory (see e.g. infra nn. 87, 96, 253) and they lacked the wit to ask someone who did to read their manuscript. Our commentary seeks to remedy this defect.

5. The mistranslations usually derive from the fact that the editors do not understand what Nietzsche is writing about. For example we have "he got involved in the Hemocopides [sic] trial" (GBP 173) instead of "he became involved in the trial concerned with the mutilation of the herms". Or "born in the Paeania province of Athens" (GBP 179) for "born in the Athenian deme Paeania".

Our text is based on an enlarged photocopy of the original manuscript in the Weimar archive. This copy was provided us by Professor David J. Parent (Illinois State University at Normal, Illinois). Without this copy our edition would have been impossible. We wish to stress the magnanimity of Professor Parent, who supplied us with the documents which he knew would be used to correct an edition for which he was in part responisible. Amicus Plato, sed magis amica veritas. 


\author{
II. \\ Text and Commentary ${ }^{6}$
}

Anhang: Abriss der Geschichte der Beredsamkeit

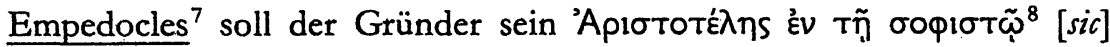
фnoıv La. ${ }^{9}$ VìII 57, wie Zeno der Erfinder der Dialektik ${ }^{10}$ : ein grosser Volksredner im höchst bevölkerten Agrigent, der eine Demokratie dort einführt ${ }^{11}$. Der Syracusaner Corax hat eine Téx $v \eta$ hinterlassen: der Redner müsse nach dem cikós, dem Wahrscheinl〈ichen〉, streben: er unterscheidet

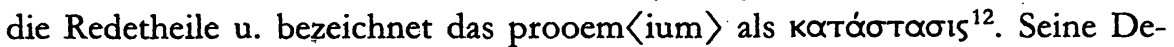

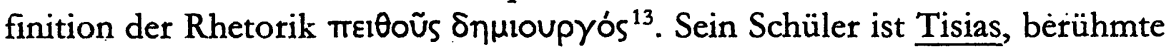
Geschichte, dass er ihm, nach einem Pact, erst den Lohn zahlen wolle, wenn er den ersten Prozess gewonnen habe (dieselbe Gesch〈ichte〉 von Protagoras u. Euathlos ${ }^{14}$ ). Korax [sic] verklagt ihn u. stellt den Satz auf, dass er jedenfalls das Geld erhalten müsse, im Fall des Sieges nach dem Spruch des Gerichts, in dem des Unterliegens nach dem Pakt. Tisias dreht es um: er habe in keinem Falle zu zahlen: siege er, weil ihn der Richterspruch dann entbinde, siege er nicht, so finde der Contrakt keine Anwendung. Die Richter jagen

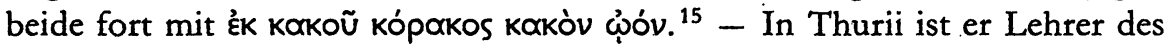
Lysias ${ }^{16}$, in Athen des Isocrates ${ }^{17}$ gewesen, c. 480 geb., herumwandernd, wie

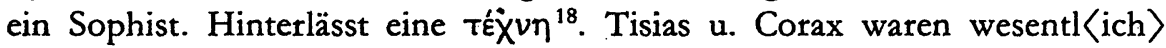
Lehrer des Prozessirens. Anders steht es mit den Sophisten des eigentl〈ichen $\rangle$ Griechenlands u. der östl〈ichen〉Kolonien, bei denen der eig〈entliche〉 Name бофıбтn's aufkommt. Sie geben eine enkyklopädische [sic] Bildung. Protagoras v. Abdera, geb. 485 , durchzog etwa von 455 (von wo die Sophistik zu datiren ist) die hell<enischen〉 Städte. Auf die attische Beredsamkeit hatte Pro-

- We have silently standardized punctuation and have omitted periods where abbreviations are expanded. Bars indicate the beginnings and ends of manuscript pages.

- For Empedocles und Corax see F. Blass, Die attiscbe Beredsamkeit, I. Von Gorgias bis qu Lysias. Leipzig, 1868, 17-20 and Empedocles frag. 1 Radermacher (henceforth: R). Blass I was until Lysias Nietzsche's principal source, and often is simply copied.

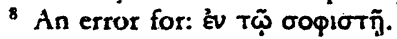

"GBP 166 read "2a.", because they cannot recognize the abbreviation for Diogenes Laertius, one of Nietzsche's favorite authors.

") Aristotle frag. 65 Rose in Diog. Laert. 8. 57.

$"$ Diog. Laert. l.r.

12 Frag. $24 \mathrm{R}$.

${ }^{13}$ Frag. 13 R.

1480 A 1 (11. 255. 12-14) D-K': see Plat. Pbaedr. 272 B-C.

${ }^{15}$ See Corax and Tisias frag. $6 \mathrm{R}$ with commentary. Nietzsche's source is Blass 1, 20.

16 Frag. $3 \mathrm{R}$.

17 Frag. 5 R.

${ }^{18}$ Frag. $10 \mathrm{R}$. 
tag〈oras〉 viel früher Einfluss als die Sikelioten. Er verheisst zu lehren tòv

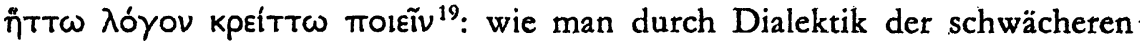
Sache den Sieg schaffen könne. Diese Dialektik sollte alle anderen Künste u. Wissenschaften entbehrlich machen: wie man den Geometer, ohne Geometer zu sein, niederdisputiren könne: so über die Naturphilos〈ophie〉, den Ringkampf, das prakt<ische〉Staatsleben ${ }^{20}$. Die Schüler mussten Musterstücke

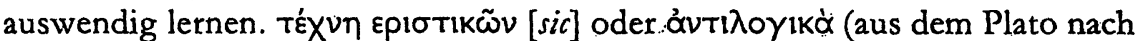
Aristoxenus den Stoff zur Politeia entnommen haben soll ${ }^{21}$, dh. Tाрі тоũ Sikaíov). Auch die anderen grossen Sophisten kommen in Betracht. Als praktisches Ergebniss dieser neuen Bildung nach der Mitte des fünften Jahrhunderts: der grosse Pericles. Er disputirte viel mit Protagoras ${ }^{22}$ : Plato leitet die höchste Meisterschaft in der Beredsamkeit aus der anaxagorischen Philosophie her: sie verlieh dem Geiste einen erhabenen Flug u. verstehenden Blick in das Innere der Natur u. des Menschen. Phaedr. p. 269 E. Damals schämten sich noch die mächtigsten Männer, in den Städten Reden zu verfassen u. Geschriebenes zu hinterlassen ${ }^{23}$. Es fehlte dem Perikles noch ganz die leidenschaftl〈iche〉 Form der späteren Redner, besonders des Demosthenes. Unbewegt stand er da, der umgewickelte Mantel bewahrte den gleichen Faltenwurf, der hohe Ernst der Mienen verzog sich nie zum Lächeln, die Stimme behielt dieselbe Höhe u. Stärke - alles undemosthenisch, doch wundersam imponirend ${ }^{24}$. - Zuerst bildet Gorgias in Sicilien einen künstlerischen Stil für prosaische Rede aus, der freilich seine Anwendung in Lobreden, nicht in praktischen Reden fand, u. bringt diesen nach Athen. In Athen entwickelt sich nur wenig später, aus der gleichfalls eingeführten Rhetorik des Tisias u. der Dialektik der östlichen Sophisten, in Anlehnung an die bestehende Sitte des 'Advokatenthums, die dem Prozessirenden die-

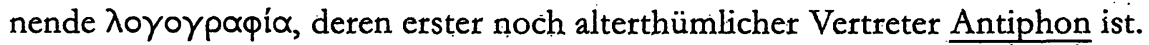
Auch sein Stil macht den Anspruch, ein künstlerischer zu sein: daher die Veröffentlichung der geschriebenen Reden, als Muster für die. Nachahmung.

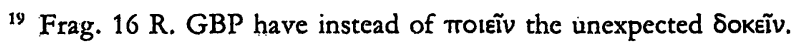

20 Plat. Soph. 232 C-E: For Protagoras see Blass I, 23-29.

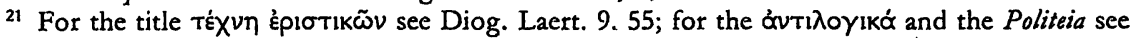
Diog. Laert. 3. 37.

22 Protagoras frag. $15 \mathrm{R}$ (Plut. Per. 36. 5).

23 Plat. Phaedr. 257 D.

${ }^{24}$ Plut. Per. 5. For Perikles see Blass I, 33-37. Compare Blass I, 37: "Unbewegt, wie ès uns beschrieben wird, stand er da, der umgewickelte Mantel bewahrte den gleichen Faltenwurf; der hohe Ernst der Mienen verzog sich nie zum Lächeln, die Stimme behielt stets gleiche Höhe und Stärke. Eben dies, was mit der demosthenischen Rede unvereinbar ist und auch in den Worten alle gewaltsame Leidenschaft ausschliesst, machte auf die Zuhörer, wie Plutarch berichtet, einen wundersam mächtigen Eindruck." Here and often elsewhere the similarity of language proves that Nietzschẹ's source was Blass I. He did not consult the ancient sources directly. 
Weiter bildet der Rhetor Thrasymachus den der praktischen Rede angemessenen Stil, indem er an die Stelle von Gorgias' Prunk u. Antiphon's steifer Würde die gerundete Periode $\mathrm{u}$. den gebildeten Ausdruck setzt ${ }^{25}$. Mitten in dieser Bewegung stehen Männer wie Kritias und Andokides, die selbst keine Sophisten sind. Endlich Lysias ${ }^{26}$, der zweite grosse Logograph: er geht noch weiter als Thrasym〈achus〉 u. wendet ganz den Ausdruck des gemeinen Lebens an ${ }^{27}$, den Periodenbau u. den Schmuck der Figuren wohl kennend, doch nicht überall verwendend. Alles dies in einem Zeitraum von 30 Jahren: in denselben entstehen schon neue Richtungen, die des Isocrates. - Also Antiphon aus Rhamnus, zur Zeit der Perserkriege geboren, etwas jünger als Gorgias: der erste, der Reden für andere verfasste: er hatte den Beinamen Nestor als Redner, zur Auszeichnung ${ }^{28}$. Völliger Mangel an polit〈ischem〉 Ehrgeiz. Grosses Lob, Thucyd. 8, 63 [sic $]^{29}$, viell〈eicht〉 sein Schüler.|

AA. ${ }^{30}$ Die Anordnung der Rede sehr regelmässig. Von Isäus ab, in raffinirter Zeit, liebt man die Kunst entgegen der Natur anzuwenden ${ }^{31}$. Würdevoller Ausdruck, der öffentl<iche〉 Sprecher musste damals gemessen auftreten, eine etwas entfernte Sprache sprechen ${ }^{32}$. Der erhabene Stil ist natürlich dem schlichten des Lysias weit näher als der erhabene Stil in der Geschichte

25 Dion. Hal. Isae. 20 (123. 10-12 (627 f.) Usener-Radermacher (henceforth: U-R)) = frag. $10 \mathrm{R}$.

26 Without informing the reader GBP 174 transfer "Endlich-Isocrates" to the later discussion of Lysias. They wish to put together all passages fhat concern each author, assuming that this was the intention of Nietzsche himself. Here they fail to understand the adverb 'endlich'. With 'endlich' Nietzsche's first version reaches a temporary conclusion. The sentence "Endlich [ist] Lysias, der zweite grosse Logograph:" in the position where GBP 174 have moved it is only tautological. After having treated Lysias' character as a logographer in a rather detailed form, the expression "Finally Lysias is the second great logograph" (GBP 175 ) is superfluous. Moreover, GBP do not see the reference to what was said earlier of Thrasymachus. Nietzsche's order must be restored.

${ }^{27}$ Dion. Hal. Demosth. 2 (130. 6-10 (956) U-R).

28 [Plut.] $832 \mathrm{E}$.

29 Nietzsche, followed by GBP, apparently reads 63 . The correct reference is 68 .

30 The abbreviation could stand for 'Anmerkung' or, more probably, for Antiphon. The following passage is written on a. separate sheet only half filled. The order of the pages can not be exactly reconstructed and the precise method of composition is not knawn: see infra $\mathrm{nn} .52$ and 75 . The reconstruction here is only exempli gratia. The context concerns Antiphon.

31 An almost litcral transcription from Blass 1, 112: "Hieraus geht denn im allgemeinen hervor, dass die Anordnung bei Antiphon eine durchaus natürliche und einfache ist. Denn was die einzige Abweichung von der gewöhnlichen Reihenfolge, die Einschaltung einer Argumentation vor der Erzählung, anbetrifft, so ist dieselbe erstlich für Antiphon nicht ungewöhnlich, sondern regelmässig, zweitens aber ist das, was in diesem Theile bchandelt wird, stets der Art, dass es hier und nirgends anderswo seine natürliche Stelle hat. Jene älteren Redner ich meine die bis zu Lysias und Isokrates eingeschlossen - waren noch nicht darauf verfallén, auch entgegen der Natur die Kunst anzuwenden, wic es die spätcren von Isäos ab, als das Zeitalter raffinirter geworden war, zu thun pflegten".

32 Sec Blass 1, 113. 
oder der Tragödie ${ }^{33}$. Alterthümlichkeit gesucht als Mittel zur Würde: während schon Perikl〈es〉 dem modernen Dialekt folgt, das ältere $\xi^{u} v u$ u. $\dot{k}^{34}, \sigma \sigma$ für

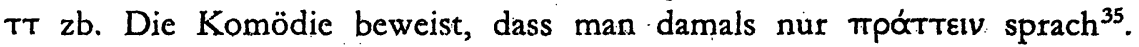
Andok〈ides〉, Lysias usw. schliessen sich der neuen Ausspr〈ache〉 an, nicht

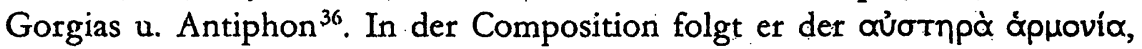
entgegen der $\gamma \lambda \alpha \phi u p \alpha \dot{\alpha}$ des Isocrates ${ }^{37}$. - Thucydides ${ }^{38}$ erwählte, nach Dionys〈ius $\rangle$, anstatt der gewöhnlichen u. schlichten Redeweise die übertragene, alterthümliche, fremdartige: anstatt der glatten Composition die rauhe $u$. kraftvolle: dann vielfache u. ungewöhnl〈iche〉 Gestaltung der Worte u. Glieder: endlich mit wenigen Worten möglichst viel auszudrücken ${ }^{39}$. Als

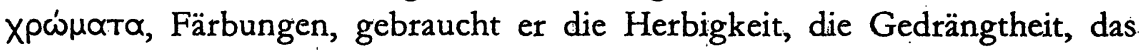
Gewichtvolle, das Erschreckende, das Pathetische ${ }^{40}$. Dionys〈ius〉 sagt: wenn es ihm gelinge, diesen Charakter nach Wunsch darzustellen, so seien seine Erfolge fast übermenschlich. Doch mache die Kürze der Rede dunkel, u. das Künstliche steht nicht immer am rechten Orte u. im rechten Masse ${ }^{41}$. Thucyd〈ides〉 erschreckt, wo Lysias u. Herodot, angenehm berühren: Lysias bewirkt ein Nachlassen, er ein Anspannen, jener schmeichelt sich ein u. überredet, dieser zwingt. Denn wie Herodot, Meister im Ethos, er im Pathos. Die Schönheit des Herodot ist eine heitere, die des Thucyd〈ides〉 eine furchtbare ${ }^{42}$. - Thrasymachus, ein Altersgenosse des Lysias, in der Einlei-

${ }^{33}$ See further Blass I, 114: "So ist der erhabene Stil in der Beredsamkeit dem schlichten des Lysias und Isäos weit mehr genähert, als in der Geschichtsschreibung, oder in der Tragödie der Abstand der entgegengesetzten Gattungen ist."

${ }^{34}$ In superscript. The archaic gưv und ḱs were replaced by oúv and eis in later Attic.

${ }^{35}$ Compare Blass I, 114: "Würde und Alterthümlichkeit sind nahe verwandte und zusammengehörende Dinge; denn wenn jene in der Entfernung von der gemeinen Sprechweise besteht, so muss auch der Gebrauch veraltender Formen der Rede Würde und Feierlichkeit verleiken. Wir können uns daher nicht wundern, wenn Antiphon gleiçhwie Thukydi les nicht den Dialekt jener und der späteren Zeit, sondern den der früheren gebraucht, was namentlich in

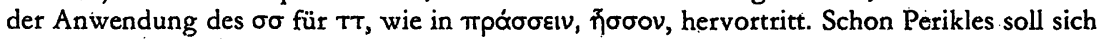
in seinen öffentlichen Reden dem modernen Dialekt anbequemt haben, und jedenfalls giebt uns die Komödie vollgültiges Zeugniss, dass man zu Antiphon's Zeit durchaus so und nicht anders sprach." Again here GBP do not understand the subject matter. They read: "weiss es schon Perikles: छúv, és. Dem modernen Dialekt folgt das ältere $\sigma \sigma$ für $\tau \tau, z$. B. Die

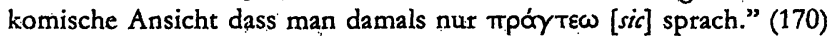

${ }^{36}$ See Blass I, 114, immediately after the last citation.

37 Dion. Hal. De comp. verb. 22 and 23.

${ }^{38}$ In the case of the two separate pages (see infra nn. 52, 75) GBP remove the part concerning Thucydides and make a footnote of it (199 n. 14). Presumably they are unaware that the historian Thucydides is stylistically a rhetor. Moreover, there are many famous speeches in his work.

39 Dion. Hal: Tbuc. 24 (361, 4-14 (866 f.) U-R); see Blasss I, 204.

40 Dion. Hal. Thuc. 24 (363. 12-15 (869 f.) U-R); see Blass I, 204.

${ }^{41}$ Dion. Hal. Thuc. 24 (363. 18-24 and 364. 1 f. (870) U-R); see Blass I, $204 \mathrm{f}$.

${ }^{42}$ Dion. Hal. Ad Cn. Pomp. 775 ff, and Demosth. 2 (131: 3-11 (957f.) U-R). Again Nietzsche used Blass and not the ancient sources: see Blass I, 223: "Thukydides erschreckt, wo jene 
tungsscene der platon〈ischen〉 Republik hervortretend, als Karikatur, anmasslich, käuflich, unsittlich, dummdreist ${ }^{43}$. Er ist überwiegend Techniker ${ }^{44}$ : er ist der Begründer der mittleren Gattung des Stils ${ }^{45}$, er ist Erfinder der für praktische Zwecke passenden Periode ${ }^{46}$, endl〈ich〉 hat er zuerst den päonischen Rhythm $\langle$ us $\rangle$ (nach Aristot $\langle\text { eles }\rangle^{47}$ ) angewandt ${ }^{48}$. Also er erfindet die

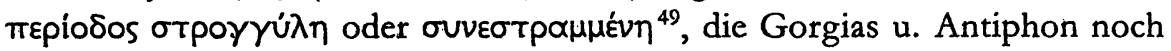
nicht kennen. Der Gedanke wird zu einer Einheit zusammengedrückt: bei Gorgias reiht sich lose Antithese an Antithese. Nach Cicero hat er fast allzu rhythmisch geschrieben ${ }^{50}$. Er ist bahnbrechend für die späteren praktischen Redner, bes〈onders〉 Lysias, im Gegensatz zu dem panegyrischen Isocrates ${ }^{51}$.|

Der ${ }^{52}$ berüchtigte Critias ist sehr ausgezeichnet: es muss auffallen, dass er nicht die Stelle des Andocides im Kanon bekam, aber ihm schadet, einer der Dreissig gewesen zu sein: Würde in den Gedanken, Einfachheit in der Form. Er ist Vertreter des jungen Atticismus. Wenig Schwung ${ }^{53}$ u. Feuer.

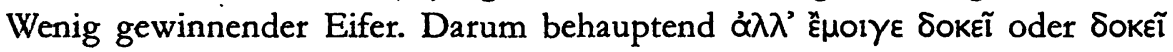
$\delta^{3}$ ênoı $\gamma \varepsilon^{54}$. Andocides kein Meister der praktischen Rede, dazu fehlt ihm allerseits Durchsichtigkeit. Sehr wenig Schmuck, wenig Lebendigkeit ${ }^{55}$. Sein

angenehm berühren; Lysias bewirkt ein Nachlassen, der Geschichtschreiber [sic] fordert ein Anspannen des Geistes, dieser zwingt, wo jener sich unvermerkt einschmeichelnd überredet: Jener ist, wie Herodot, im Ethos, Thükydides im Pathos Meister; die Schönheit des Herodot ist eine heitere, die des andern eine furchtbare".

43 "dreist" stands in superscript.

4 Plat. Rep. 1, $336 \mathrm{ff}$.

45 After Theophrastus mepi $\lambda \dot{\varepsilon} \xi \varepsilon \omega s$ frag. IV Schmidt, cited in Dion. Hal. Demosth. 3 (132. 3-7 ( $958 \mathrm{f.}$ ) U-R).

46 So Theophrastus mepì $\lambda \hat{\xi} \xi \varepsilon \omega s$ frag. 111 Schmidt, cited in Dion. Hal. Lys. 6 (132. 3 (958 f.)

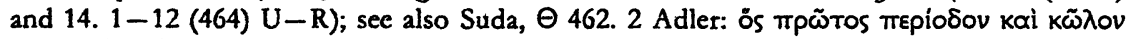

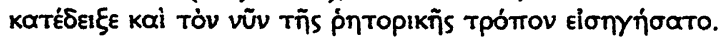

4: Rhet. 1409 a 2 = frag. $12 \mathrm{R}$.

48 See Blass I, 246.

49 Thrasymachus frag. 15 R. See Suda $\Theta 462$ Adler.

50 Cic. Orat. 175: Nam neminem in eo genere scientius versatum Isocrate confitendum est, sed princeps inveniendi fuit Thrasymachus, cuius omnia nimis etiam exstant scripta numerose."

51 There are many almost literal citations taken by Nietzsche from Blass I, 248-250.

52 The text "Der berüchtigte Critias" up to "Lysias" is on a separate page only three quarters filled. It is a short passage covering Critias to Lysias that does not fit easily into the main narrative. It is difficult to reestablish the order of the sheets. As far as can be deduced from a number on the top this page was inserted after the first loose one. Why GBP put Critias before Antiphon, remains obscure. The sequence Antiphon - Thucydides - Thrasymachus - Critias - Andocides - Lysias derives from Blass 1.

53 GBP 170 read: "Wenig Sprung und Feuer." But see Blass I, 267: "denn Feuer und Schwung fehlt auch in den Poësien" and the parallel in the 1874 lecture notes (ap. cit. supra n. 2), p. 209.

54 88 B 46 D-K'. Sce Blass I, 265.

s See Blass I, 290: "W'eiter zeige er sehr wenig Schmuck; elsensowenig rednerische Lebendigkeit". 
Stil ist nicht gleichmässig, sondern buntscheckig ${ }^{56}$. Mit Entlehnungen von selbst tragischen Wendungen. Im Allgemeinen die Sprache des gewöhnl〈ichen〉 Lebens ${ }^{57}$. In der argumentatio ist er mittelmässig, im Erzählen von Geschichten 〈geschickter ${ }^{58}$, weshalb er die Reden auch zumeist mit Erzählungen füllt ${ }^{59}$. In der Ausprägung des Ethos ist Lysias viel vorzügl〈icher〉, im Pathos beide schwach. Unter den Zehnen ist er der geringste an Talent $u$. Studium: obgl〈eich〉 es eine grosse Ehre ist, überhaupt unter sie aufgenommen $z u$ werden ${ }^{60}$. - Lysias. Im platon〈ischen〉 Phaedrus wird die lysian〈ische〉 Rede scharf getadelt, die Sache sei unphilosophisch angefasst ${ }^{61}$ : dem Isocrates wird dagegen eine gewisse philosophische Richtung nachgerühmt: Verschiedenheit der Stoffe beider, da Lys〈ias〉 ausser erotisch〈en〉 Kleinigkeit〈en〉 nur die von Plato verachteten Prozessreden, Isocrates epideiktische Reden mit polit〈ischen〉 Hintergründen schrieb, mit mehr idealen als praktischen Gesichtpunkten $[s i c]^{62}$. Aber es ist nicht gerechtfertigt; die Bevorzugang allein aus dem Stoffe zu begreifen: Perikles wird als grosser Redner anerkannt. Das. Fehlen allgem〈einer〉. Begriffsbestimmungen, sehr selten allgemeine Principien berührt ${ }^{63}$. Dann fehlt die richtige Ordnung $u$. logische Folge der Gedanken. Die kunstvolle Rede dürfe nicht eine Häufung von Argumenten sein, die beliebig so u. so gestellt werden könnten ${ }^{64}$. Plato lobt allein den Stil: sowohl klar als abgerundet ${ }^{65}$. Später, bes〈onders〉 bei Dionys〈ius〉, ist er der Vertreter des Xapaktì $\alpha \dot{\alpha} \varepsilon \lambda \eta \zeta^{66}, \sigma u v \varepsilon \sigma \pi \alpha \sigma \mu \dot{\varepsilon} v \eta^{67}$. Cicero gebraucht von solchen Rednern die Aus-

${ }^{56}$ See Blass 1, 291: "Sein Ausdruck erhält auf diese Weise etwas buntscheckiges".

57 See Blass I, 291: "Im allgemeinen ist, wie gesagt, Andokides' Sprache die.des gewöhnlichen Lebens".

58 Compare Blass I, 292. GBP's 〈viel besser> must be discarded.

59 See Blass I, 292: "Andokides ist in der Argumentation mittelmässig, im Erzählen dagegen zeigt er entschieden Geschick und füllt deswegen auch seine Reden grösstentheils mit Erzählungen."

6) See Blass I, 299: "Man wird also im ganzen sich dem Urtheile O. Müller's [Gescbichte der griechischen Litteratur bis auf das Zeitalter Alexanders II, Breslau, 1841, $\left.{ }^{2} 1856,339\right]$ anschliessen können, dass Andokides unter den Zehnen der geringste an Talent und Studium sei; obwohl es ja ein grosser Ruhm ist, überhaupt mit einigem Rechte in diese Liste aufgenommen zu sein."

61 Plat. Phaedr. 279 A after Blass I, 375.

62 See Blass I, 375: "so kann man zunächst noch immer an die Verschiedenheit der Stoffe beider denken, da Lysias ausser erotischen Kleinigkeiten fast nur die von Platon geringgeschätzten Prozessreden, Isokrates hauptsächlich epideiktische Reden politischen Inhalts schrieb, mit mehr idealen als praktischen Gesichtspunkten".

${ }^{63}$ See Blass I, $375 \mathrm{f}$.

of See Blass I, 376.

65 Plat. Phaedr. 234 E after Blass I, 377.

6 Dion. Hal. Dem. 2 after Blass I, 379 n. 3.

67 Dion. Hal. Dem. 15 after Blass I, 379 n. 3. 
drücke tenues, acuti, subtiles, versuti, humiles, summissi ${ }^{68}$. Die künstlerische Nachbildung ${ }^{69}$. der gewöhnl〈ichen〉 Rede, anscheinend für jeden leicht, sei ausserordentlich schwer. Der tenuis kann bloss belehren ${ }^{70}$ : die Leidenschaften zu erregen, gestattet ihm die Beschränktheit der Mittel nicht. Darum ist Lysias nicht der vollkommene Meister, sondern Demosth $\langle e n e s\rangle^{71}$, der je nach den Umständen bald knapp, bald gewaltig, bald gemässigt spricht ${ }^{72}$. Das Streben nach wahrhaft. Grossem ist mit einem theilweisen Misslingen verbunden, während kein grosses Genie dazu gehöre, sich bei niedriger gerichtetem Streben von Fehlern fernzuhalten. Auch erhebe sich jener oft Strauchelnde, doch sein hohes Ziel Erreichende weit über diesen ${ }^{73}$ : die Grösse der Vorzüge, nicht die Zahl entscheide. So der Autor mepi üyous bei einem Vergleich von Plato u. Lysias ${ }^{74}$.

Das Alterthum hatte 60 Reden von $\mathrm{ihm}^{75}$, Caecilius erklärte davon 25 für

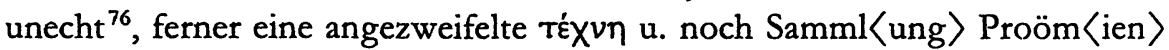
u. Epiloge. Uns sind 15 erhalten, wovon $4^{77}$ zu Tetralogien dreimal zusammengestellt sind. Cf. Blass p. $91^{78}$. Meist sind es $\lambda o ́$ yol фovıkoí, dh. in Criminalfällen, nur 3 beziehen sich auf wirkl〈iche〉 Fälle. Er gilt neben Thucyd〈ides〉 als der Meister der alterthümlich strengen Redekunst ${ }^{79}$. Besonders wichtig für den politischen Stil der Reden. Der erste im Canon der 10 att〈ischen〉 Redner, den man viell<eicht〉 in Alexandria anlegte.

Andocides geb. $468^{80}$, vornehmes Geschlecht, die Würde der Mysterienherolde für die Eleusinien vererbt ${ }^{81}$. Bewegtes politisches Leben während des pelop〈onnesischen〉 Kriegs, als Heerführer u. Gesandter ${ }^{82}$, verwickelt in den

${ }^{68}$ Particularly in the Orator after Blass I, 379 n. 3.

60 See Blass I, 381. GBP 174 read: "Doch eine straffe Nachbildung".

$\because$ Cic. Orator 76 after Blass I, 380.

1 Dion. Hal. Dem. 15 after Blass I, 381 n. 1.

72 Cic. Brut. 35 after Blass I, 381 n. 3.

i3 GBP 174 read: "Auch so erheben sich nun oft Versuchende, doch kein hohes Ziel Erreichende weit über das Mass."

${ }^{-4}$ Ps.-Long. de subl. 32. 8. Nietzsche closely follows Blass I, 382.

${ }^{75}$ Not Lysias, but Antiphon is meant. After the two separate sheets inserted here the text returns to the bottom of the first page.

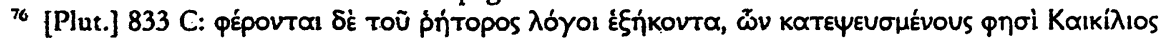

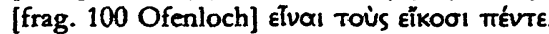

$\pi$ "3 $\times 4$ " deleted to 4 .

76 Blass 1,91 . Nietzsche cites only here his main source for the first part of his Abriss.

${ }^{79}$ For the comparison of Antiphon with Thucydides see Dion. Hal. de comp. verb. 10 (36.

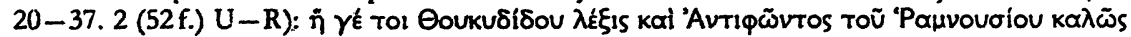

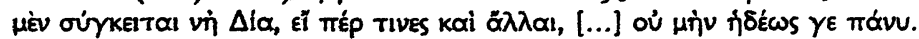

in For the date of. Andocides' birth see Blass I, 271. Blass regards the date of $468 / 7$ reported in his vita as "Resultat eines falschen Sch'usses", based on the misunderstood report "von des Andokides' Feldherrnschaft auf Samos". Blass claims that Andocides was born c. 440.

${ }^{81}$ For the context see [Plut.] $834 \mathrm{C}$.

22 Thuc. 1. 51. Andoc. 3. 6. This must be the elder Andocides; see Blass 1, 270. Nietzsche ignores the genealogy. 
Hermokopidenprozess ${ }^{83}$. Wir haben 4 Reden in eigenen Angelegenheiten,

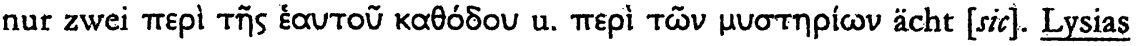
geb. $459^{84}$ zu Athen, begüterte Eltern, die ihre Heimat Syrakus 475 verlassen u. als Metöken in Athen sich niedergelassen. Er nahm an der Gründung von Thurii 444 theil, lernt dort unter Tisias u. Nikias Rhetorik, kehrt 412 nàch Athen zurück, stirbt 377 . Seinen Ruf begründet die Rede gegen Eratosthenes, den Mörder seines Bruders Polemarchus, einer der $30^{85}$. Er stiftet eine wichtige Redeschule. Von 425 Reden hielten' die Alten 230 für echt ${ }^{86}$. Von

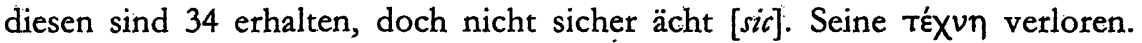
Meister des tenue dic $\langle$ endi $\rangle\left\langle\right.$ $\langle\text { enus }\rangle^{87}$ u. der geticht $\langle$ ichen $\rangle$ Beredsamk $\langle$ eit $\rangle$. Isocrates $^{88}$ geb. $436 \mathrm{zu}$ Athen, Schüler des Tisias, Gorgias, Prodikus u. Socrates, der grösste Lehrer der Bereds〈amkeit〉. Schüchtern, schwache Stimme. Sein Vater hatte Ende des peloponn〈esischen〉 Kriegs sein Vermögen verloren: er schrieb Reden daher. 392 eröffnet er die Schule; zuerst auf Chios mit 9 Schülern ${ }^{89}, 388$ in Athen mit 10090, höchst ruhmvoll. Er starb freiwillig (durch Hunger) 338 nach der Schlacht bei Chäronea, aus Schmerz über den Verlust der Freiheit ${ }^{91}$. Mächtiger patriot〈ischer〉Charakter, ideales Streben. Führt die Schule zur polit〈ischen〉 Bereds〈amkeit 〉. Von 60 Reden erkannte Caecil〈ius〉 28 als ächt $[s i c]^{92}, 21$ erhalten, 15 panegyrische davon u. 6 gerichtl〈iche $\rangle$. Am berühmt〈esten〉 der Panegyr〈ikos〉382, in

83 Thuc. 6. 60.

${ }^{84}$ Here too Nietzsche is poorly informed, although Blass I, 336 takes a vehement position against 459 as the date of birth. The ancient source for 459 is based on the erroneous belief that Lysias came to Thurii in the year when the colony was founded (444); see K. J. Dover, Lysias and the Corpus Lysiacum, Berkeley/Los Angeles, 1968, 38-42 and A. Lesky, Gesch.d. griech. Lit., Bern/München, ${ }^{3} 1971,665$. Nietzsche may already base his dates and numbers on Westermann I (see infra n. 88), who also has 458 (p. 73).

${ }^{85}$ Here are meant the thirty tyrants, who formed the government after the Athenian defeat of 404 until the restoration of democracy by Thrasybulus in 401 . Eratosthenes was part of this oligarchic regime.

${ }^{86}$ [Plut.] 836. A. Blass I, 345 mentions 233 genuine speeches, taking the three additional ones from Photius.

87 GBP 172 read tenue dia[poreticum] g[enus], whatever that means.

88 The most recent handbook for Greek oratory in 1873, Blass I, ended with Lysias: Blass II and III were published later, 1874-1880. Therefore, Nietzsche had to resort to Blass' predecessor; A. Westermann, Geschichte der Beredtsamkeit in Griechenland und Rom, I. Geschichte der griechischen Beredtsamkeit, Leipzig, 1833. As will be shown, Nietzsche follows the same method he used with Blass, simply excerpting Westermann for the period not included in Blass I. For Isocrates see Westermann I, 77-83.

89 See [Plut.] 837 B. "Mit 9 Schülern" stands as a superscript in simall letters. See Westermann I, 79 n. 6.

* I. e. Schülern. "Mit 100" stands as a superscript. See [Plut.] 837 Cff. and Westermann I, 79 n. 7.

1 [Plut.] $837 \mathrm{E}$ und $838 \mathrm{~B}$.

${ }^{92}$ Caecilius frag. 117 (103. 22-24) Ofenloch. 
seinem 94. Jahre geschrieben ${ }^{93}$. Nicht sicher die TÉ $\chi \cup \eta$. Seine talentvollsten Schüler Theopomp, Ephorus, Philiscus, Androtion, dann Isaeus, Demosth〈enes〉, Hyperides, Theodectes usw ${ }^{94}$. Er ist ein Feind alles Sophisten-

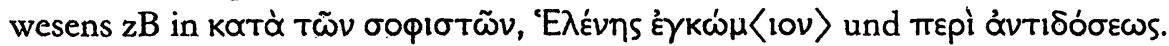
Erst im hohen Alter wendet er sich der Theorie zu, so tadelt er in $k\langle\alpha \tau \dot{\alpha}\rangle$

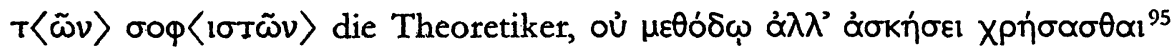
war in früheren Zeiten sein Satz. Er wendet sich zuerst zur grossen politischen

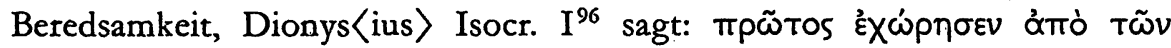

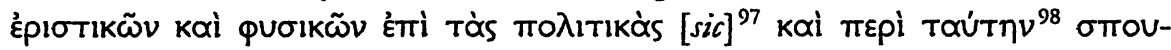

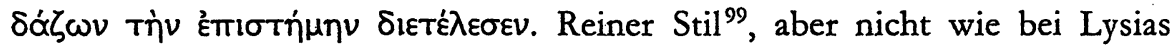

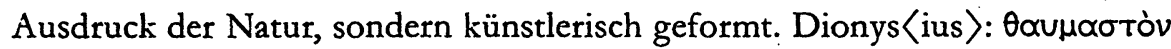

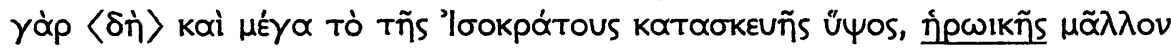

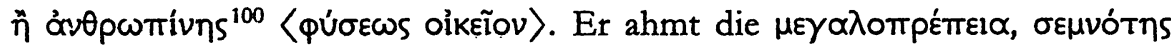
u. $\kappa \alpha \lambda \lambda_{i} \lambda$ oyi $\alpha$ des Thucyd〈ides〉 und Gorgias nach. Aber zu der erhaben〈en〉 Redegewalt des Demosth〈enes〉 verhält er sich nur wie ein Athlet zu einem Vaterlandsvertheidiger (wie Cleochares aus Myrlea gesagt hat) ${ }^{101}$. Er besitzt mehr Eleganz als Grazie, mehr Pracht als Anmuth ${ }^{102}$, Dionys〈ius〉

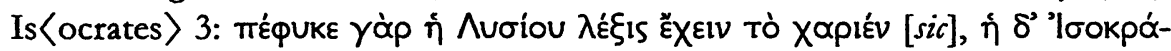
Tous $\beta$ oú $\lambda \varepsilon \tau \alpha{ }^{103}$. Gerundete Periode, viel [sic] Figuren, oft durch Überfülle monoton u. weitschweifig ${ }^{104}$. Höchst ausgearbeitet u. Lysias weit in Anord-

93 〈und der Panathenaikos). Nietzsche made a careless or even ignorant mistake. Not the Panegyrikos, but the Panathenaikos, his last speech, was written when he was 94 years old.

94 See Westermann I, 83.

${ }^{95}$ [Plut.] $838 \mathrm{~F}$ after Westermann 1,79 n. 11.

* 55. 12-14 (536). U-R. GBP 176 read Dionys. Laert; they cannot distinguish between Dionysius Halicarnaseus and Diogenes Laertius.

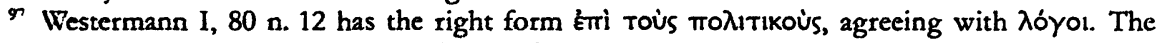
error reveals Nietzsche's unsure knowledge of Greek.

9 Nietzsche accepts F. A. Wolfs emendation after Westermann I, $80 \mathrm{n}$. 12. The manuscripts have oủrìn.

9 Dion. Hal. Isocr. 2 (56. 14-16 (537) U-R); Lys. 2 (9. $20 \mathrm{f}$. (455) U-R).

${ }^{100}$ Dion. Hal. Isocr. 3 (59. 16-18 (541) U-R). The Greek can only be understood with addition of the omitted words: see Westermann $1,82 \mathrm{n} .7$.

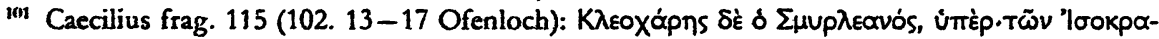

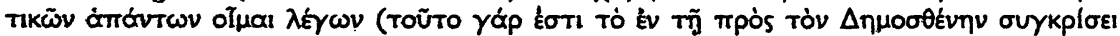

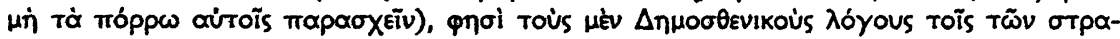

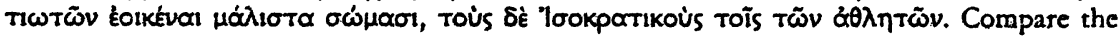
almost literal citation from Westermann 1, 81: "aber zu der erhabenen Redegewalt des Demosthenes verhālt er sich doch nur wie ein Athlet zu einem Vaterlandsvertheidiger", and Westermann 1, 83 n. 8 for Cleochares.

312 See W'estermann 1, 81: "Er besitot mehr Eleganz als wahre Grazie, mehr Pracht als Anmuth." 163 59. $11 \mathrm{f}$. (541) U-R after Westcrmann I, $83 \mathrm{n} .9$ whence the erroncous accent for $X \propto p$ plev.

tin So Westermann 1, 81: "sorgfaltig gerundeter Periodenbau und häufiges Figuriren macht seine Rede zwar wohllautend, aber of durch Ueberfülle weitschweifig und monoton." See also the ancient sources in Westermann I, $83 \mathrm{n}, 10$. 
nung $u$. Vertheilung des Stoffes überlegen ${ }^{105}$. Er hat daher für sein langes Leben nur wenig geschrieben. Übrigens mehr für den Leser als den Hörer. Wahre Gerichtsreden nur wenige oder keine, dageg〈en〉 gerichtl〈iche〉 Muster- u. Übungsstücke ${ }^{106}$. Isaeus ${ }^{107}$ lebt c. $420-348$, geb. in Chalcis ${ }^{108}$, lernt in Athen, wo er Lysias u. Isocr〈ates〉 zu Lehreen hat. Darauf wird er

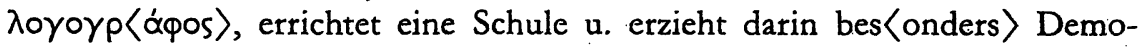
sthenes. Von 64 Reden hielten die Kritiker 50 für ächt [sic]; nur 11, alle in Erbschaftsangelegenheiten, haben sich erhalten. Technische Schriften verloren. Er gleicht Lysias in der Reinheit u. Deutlichkeit des Vortrags ${ }^{109}$ : dagegen nicht so naiv, sondern gekünstelt, gefeilt und geschmückt ${ }^{110}$; doch kräftig im Ganzen. ő

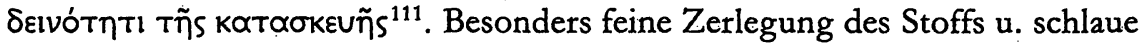
Verflechtung der Argumente: er galt als verschmitzter Betrüger bei seinen Feinden ${ }^{112}$. Der von Antiphon erfunden〈en〉, von Isocrates begründeten politischen Rede gab er schärfere Umrisse. Früher gab es nur 10 Reden. $1785^{113}$ wurde in einem cod〈ex $\rangle$ Laürent〈ianus〉 noch eine 11te, $\pi \varepsilon p i$ toũ

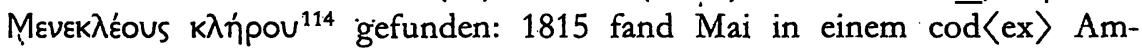

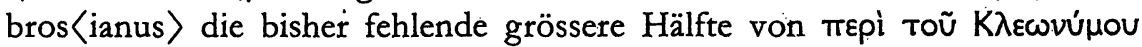

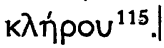

In der macedon〈ischen $\rangle$ Periode ${ }^{116}$ ist Demagog und Redner fast gleichbedeutend ${ }^{117}$. Die antimacedonische Partei Lycurgus, Demosthenes, Hyperides $\mathrm{u}\langle$ nter $\rangle$ anderen, macedonisch ${ }^{118}$ Aeschines, Eubulos, Philocrates, Demades, Pytheas. Lykurgus (der VI. ${ }^{119}$ ) aus dem alten Geschlecht der Eteobutaden ${ }^{120}$, c. Ol. $96^{121}$ geb., durch Platon u. Isokrates gebildet ${ }^{122}$, früh in

105 See Westermann I, 81.

106 So Westermann I, 81 and 83 nn. 11 and 12.

107 See Westermann I, 87-89.

${ }^{108}$ Dion. Hal. Isae. 1 (93. 2-5 (586) U-R) does not make clear whether Isaeus came from Athens, as Hermippus alleges, or from Chalcis, as Demetrius.

109 See Dion. Hal. Isae. 3 (94-95. 3 (589) U-R).

${ }^{110}$ Dion. Hal. Isae. 3 (95. 4-9 (589 f.) U-R) after Westermann I, $87 \mathrm{f}$.

11 Dion, Hal. Isae. 3 (95. 10 f. (590) U-R) after Westermann I, 89 n. 12.

112 Dion. Hal. Isae. 4 (96. 5-18 (591) U-R).

${ }^{113}$ From here to the end of the section see especially Westermann I, 89 n: 9.

114 That is Isae. 2.

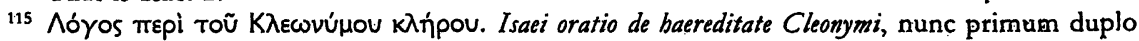
auctior, inventore et interprete Ang. Maio, Mediolani 1815 (= Isae. 1).

116 See Westermann I, 90-95.

117 See Westermann I, 93: "Demagog und Redner ist in dieser Periode fast gleichbedeutend".

118 See Westermann I, 95-100.

119 For the ten Attic rhetors Nietzsche begins to cite the numbers which Westermann used. For Nietzsche's almost literal citations concerning Lycurgus see Westermann I, $100 \ldots 103$.

120. [Plut.] $841 \mathrm{AB}$.

121 Nietzsche, after Westermann, gives the date of birth here in Olympiads. It is equivalent to c. 396. .

122 [Plut.] $841 \mathrm{~B}$. 
der polit〈ischen〉 Laufbahn. Gewissenhaft u. uneigennützig. Von $15 \mathrm{im}$ Alterthum vorhandenen Reden ist eine gegen Leocrates auf uns gekommen. Nach Dionys〈ius〉 ist die moral〈ische〉 Tendenz hervortretend. Darstellung edel u. erhaben ${ }^{123}$, aber nicht gefällig. Härte im metaphorischen Ausdruck ${ }^{124}$, Ungenauigkeit in der Vertheilung des Stoffes, häufige Abschweifung ${ }^{125}$. Er

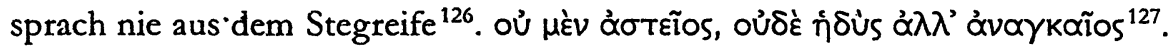
Demosthenes (VII) ${ }^{128}$ geb. im Gau Päania zu Athen 385, ist 7 Jahre alt, als Vater stirbt. Vormünder Aphobos u. Onetor ${ }^{129}$. Gebildet von Plato, Isäus u. Isokrates, 17 Jahre alt ${ }^{130}$, durch Callistratus' Rede zum Studium der Beredsamkeit fortgerissen ${ }^{131}$, ersetzt durch Eifer u. Übung, was an ihm von Natur oder durch Erziehung fehlt. 354 trat er öffentliich〉 vor dem Volkè auf ${ }^{132}$. Sein eigentlich〉 politischer Charakter entwickelt sich mit Philipp〈's〉 immer deutlicher werdenden Plänen ${ }^{133}$. Als 346 Philipp zum Frieden sich geneigt zeigte, war er.es, der unter den 10 Gesandten, die von Athen zum König geschickt wurden, fest gegen Geld blieb u. den Betrug durchschaute. Die Unterjochung von Phocis enttäuschte die Athener; Demosth〈enes〉 von zwei Übeln das kleinere wählend stimmte jetzt für den Frieden ${ }^{134}$. Philipp mischt sich bald in die peloponnes〈ischen〉 Händel: die Seele der Gesandtschaft war immer Demosth〈enes〉, der auch gegen die Staatsverräther vorging (de falsa legatione gegen Aeschines). Philipps Gewaltstreiche im Chersones ${ }^{135}$ und

${ }^{123}$ Dion. Hal. de imit. 5.3 (212. 9f. (433) U-R) (after Westermann I, 103 n. 16): 'O $\delta \dot{\varepsilon}$

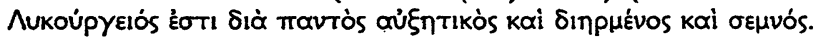

134 Hermog. 416 und Blass III 2, $99 \mathrm{f}$.

${ }_{125}$ Digressions, e. g., in the speech against Leokrates $₫ 46,83,95,98,107 \mathrm{f}$.

125 See [Plut.] $842 \mathrm{C}$. The sentence is a literal citation from note 15 in Westermann I, 103. GBP 178 read "wie" for "nie".

12. Dion. Hal. de imit. 5.3 (212. $11 \mathrm{f}$ (433) U-R) after Westermann 1, 103 n. 16. Obviously Nietzsche did not look up the original. He only copied important passages cited in Blass or in Westermann. But even so he makes a slight error: Westermann reads correctly oủ $\mu$ ìv, which Nietzsche renders oủ $\mu$ ìv.

12. See W'estermann 1, 103-113, from whom Nietzsche has copied much of his text.

12" "ist 7 Jahre $[\ldots]$ Onetor" written in superscript.

13" "17 Jahre alt" written in superscript. A further superscript, later cancelled, is illegible.

131 Nietzsche combines two traditions: 1.) the fact that aged 17 Demosthenes sued his guardians; see Liban. VIII 603. 3 Foerster. 2.) the aneedote that he heard Callistratus' speech as a young boy; see Hegesias of Magnesia (FGrHist 142 F 22) in [P]ut.] 844 B. Both are from Westermann 1,103 and 107 n. 7.

132 Nietzsche means the synegoria against Leptines.

133 Compare Westermann 1, 104: "Sein eigentlich politischer Charakter aber entwickelte sich mit Philipp's immer schärfer hervortretenden Plänen".

it Compare c.g. W'estermann 1, 104: "Erst die Unterjochung von Phocis enttäuschte die Athener; doch unter ¿wei l'ebeln das kleinste wählend stimmte jetzt auch Demosthenes für den Frieden."

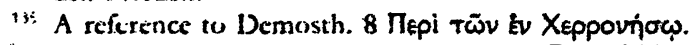


Demosth〈enes'> Anfeuerung ${ }^{136}$ führt den ruhmwürdigen Kampf in Thracien herbei, der mit dem Entsatz von Byzanz 340 endigte. Der letzte phokische Krieg rief $\mathrm{Ph}\langle$ ilipp〉 ins Herz von Griechenland, er besetzte Elatea. Bei dieser Schreckensnachricht verlor nur $\mathrm{D}\langle$ emosthenes〉 den Muth nicht, er hob, nach Theopomp, die Zahl auch der [?]gaben ${ }^{137}$. Es gelang ihm, Athen und Theben zum Kampfe zu vereinigen. Doch ohne Erfolg: mit Chäronea 338 ging Athen's Unabhängigkeit verloren. Mit Philipp's Tode 336 gab es eine neue Hoffnung: überall Empörung. Man kam zur Ruhe, als Alexander mit einem Heer erschien: nur Theben, vor Cadmea in Empörung ${ }^{138}$, wurde von Grund aus zerstört ${ }^{139}$. Athens Antheil zu strafen, verlangte Alex $\langle$ ander $\rangle$ die Auslieferung der Volkshäupter, darunter Demosth〈enes〉, doch liess er sich von Phocion u. Demades begütigen ${ }^{140}$. In der folg〈enden〉 Friedenszeit wird $\mathrm{D}\langle$ emosthenes〉 in den Prozess verwickelt, den Harpalos durch Bestechung anregte 325; ohne überwiesen zu sein, wird er verurtheilt. Er entkam u. begab sich nach Aegina. Da kam die Nachricht von Alexa〈nder〉's Tode. Leosthenes beginnt den Lamischen Krieg. Frreiwillig schloss sich $\mathrm{D}\langle$ emosthenes〉 der Gesandtschaft an, welche Athen zum allgem〈einen〉 Aufgebot an alle hell<enischen〉Staaten schickte u. wurde ehrenvoll nach Athen zurückberufen $u$. glänzend empfangen ${ }^{141}$. Aber die Schlacht bei Crannon ging verloren $322 \mathrm{u}$. Antipater zwang zum Frieden. Vor ihm, seinem Todfeinde, floh ${ }^{142} \mathrm{D}$ <emosthenes〉 nach Calauria u. dort starb er an genommenem Gift unter den Händen der Knechte des Antipater. - ${ }^{143}$ Er war von Natur nicht wie Aeschines mit mächtiger Stimme begabt oder wie Demades gewaltiger Stegreifredner ${ }^{144}$. Er musste seine Gedanken sorgfältig zusammensetzen. Seine Stimme war kurz, lispelnd, seine Gesten ohne Grazie. Es waren die gleichen Gründe, die Isocrates von der öffentl〈ichen〉 Thätigkeit

${ }^{136}$ This is an allusion to the third speech against Philipp, a masterpiece of Demosthenes' political rhetoric. Eratosthenes (FGrHist $241 \mathrm{~F}$ 32) compares his passion to Bacchic enthusiasm (Plut. Dem. 9, 850 B).

137 The MSS have Theophrastus, emended by Bünger to Theopompus (FGrHist 115 F 404). See Plut. Dem. 17, 853.E; "er [...] [?]gaben" is in superscript and therefore almost illegible. Perhaps better: "Eingaben". For this information there is no corresponding sentence in Westermann. He must have known it from Plutarch. The passage does not concern Demosthenes. Nietzsche seems to have confused Plut. Dem: $853 \mathrm{E}$ with $854 \mathrm{~B}(=$ FGrHist $115 \mathrm{~F}$ 328).

138 . The fortress of Kadmeia, situated to the south of the city, was still occupied by the Macedonians.

139 See Diod. 17. 14; Plut. Alex. 11; Arrian 1. $7 \mathrm{ff}$.

140 Plut. Dem. 23, 856 D-F.

141 Plut. Dem. 27, 858 C-859 A.

142 GBP 180 read 'flog'.

${ }^{143}$ Plut. Dem. 29-30. This dash refers to Westermann's subdivision in $\$ 56$ and $\$ 57$. But here Nietzsche seems to have used a different source.

144 Plut. Dem. 10, 850 C. 
ausschlossen. In der Gesch〈ichte〉 der Selbsterziehung. ist er höchst denk-

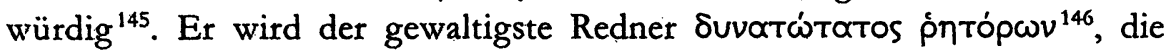

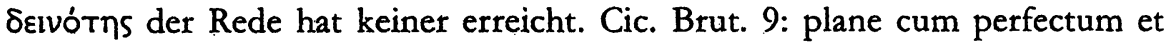
cui nihil admodum desit dixeris ${ }^{147}$. Von $65 \operatorname{Reden}^{148}$ sind $61^{149}$ erhalten,

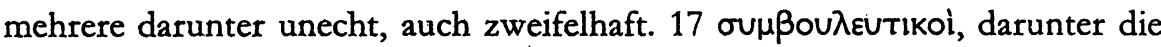
12 philippischen ${ }^{150}$. Die 7te $\pi \varepsilon p i$ 'A $\lambda$ ovvทंбov ist von Hegesipp gehalten ${ }^{151}$,

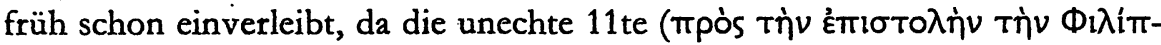
Trov) sie benutzt. Unecht auch die $42\langle$ te〉. Dagegen der Brief Philipps, der

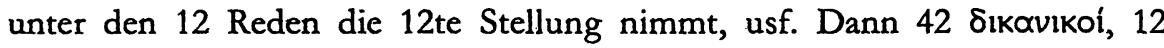
davon staatsrechtlich, 30 bürgerliches Recht. Am bedeutendsten darunter

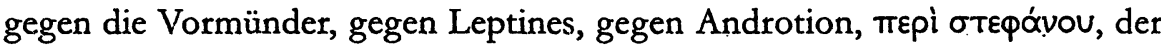

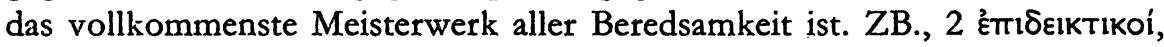

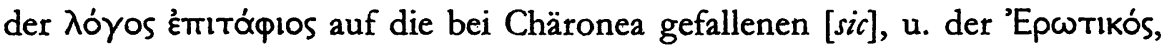
beide unecht ${ }^{152}$. Sehr zweifelhaft 56 erhalte〈ne $\rangle$ Proömien u. 6 Briefe. A. Schäfer, Demosthenes u. seine Zeit, Leipzig 1856-58, 3 Bände ${ }^{153}$.|

!Hyperides ${ }^{154}$ an kein Muster gebunden, Mitte zwischen Anmuth des Lysias u. Kraft des Demosth〈enes ${ }^{155}$. Ausdruck rein attisch, doch mit einiger Manier. Behandlung des Stoffes scharfsinnig ${ }^{156}$, doch nicht sorgfältig, vortreffl〈ich〉 in Beweisführung. Unnachahml〈iche〉 Eleganz u. Grazie ${ }^{157}$.

145 For his self-education see Blass III 1, $19-24$.

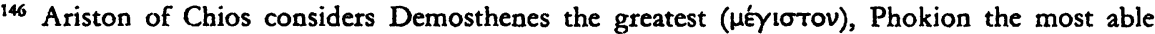

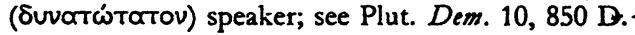

147 The citation is not strictly accurate; see Cic. Brut. 9 (35): nam plane quidem perfectum et cui nihil admodum desit Demosthenem facile dixeris.

148 [Plut.] $847 \mathrm{E}$.

149 The manuscripts give only 60 speeches; the letter to Philipp has to be excluded (6 further letters and the collection of prooemia). See Jonathan A. Goldstein, The Letters of Demosthenes, New York/London, 1968.

150 Nietzsche has 12 speeches against Philipp, because he adds the above mentioned letter to Philipp. In WS 1872 Nietzsche lectured at the Paedagogium on Demosth. Pbil. I, II: see Karl Schlechta, Nietzsche-Chronik, Munich, 1984, 39.

151 So Harpoce. I, 146. 2-3 Dindorf.

152 Dion. Hal. Dem. 23 (180. 15f.) and 44 (228. $16 \mathrm{ff}$., U-R). For the Epitaphios see also ${ }^{\circ} \dot{A}$. Schäfer, Demostbenes und seine Zeit, III, Leipzig, ${ }^{2} 1887,36$ n. 1.

153 This is only a general reference; Nietzsche did not use Schäfer as he had Blass I. Schäfer is far too detailed for his purposes. Westermann I remains his obvious source.

154 The following text from "Hyperides" to tpaxúrns is on a separate sheet, only one fourth filled. There are remarks about Hyperides, Aeschines and Deinarchos, which seem to be later notes on these men. GBP again attach them to the corresponding treatments of the various authors without indicating the transpositions. But as far as can be judged by the original page numbering, this sheet was placed here in the Abriss.

$15 s$ See Ps.-Longinus de sutl. 34. $1 \mathrm{ff}$.

iso Inter alia Dion. Hal. de imit. 5.6 (213. 3 f. (434 f.) U-R) after Westermann 1, 123 n. 9.

157 The whole short paragraph seems to be a note belonging to the larger passage concerning Hyperides (VIII): See p. 380f. The source is Westermann 1, 122: "An kein Muster streng gebunden bildet sic, selbst anmuthig und kräftig, das Mittel zwischen der Anmuth des Lysias 
Aeschines Fertigkeit im Improvisiren, seine Reden der reine Erguss eines übersprudelnden Genie's ${ }^{158}$. Kraft, Glanz ${ }^{159}$ u. Fülle. Bei aller Anmuth doch heftig u. stürmisch, zeigt mehr Fleisch als Muskel ${ }^{160}$. Sein Einfluss zeigt sich vor allem darin, dass er durch Übersiedelung der Kunst nach Asien der Stifter der verbreiteten u. mächtigen asianischen Schule ${ }^{161}$, nach dem Erlöschen der attischen, wurde ${ }^{162}$.

Dinarch $\langle u s\rangle$ ist nach Dionys $\langle$ ius $\rangle \mathrm{H}\langle$ alicarnaseus $\rangle$ nicht originell $\mathrm{u}$. hat eigentl〈ich〉 gar keinen Charakter, er ahmt bald Lysias, bald Hyperides, bald

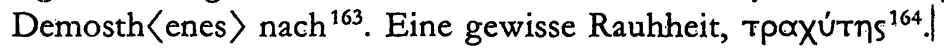

Hyperides (VIII) ${ }^{165}$ aus Athen, Schüler des Plato u. des Isocrates, Freund des Demosth〈enes〉, entging mit ihm, mit Lykurg u. Charidemus nach der Vernichtung Thebens kaum der Gefahr, ausgeliefert zu werden. Nach Alexa〈nder〉's Tode am lamischen Krieg betheiligt, wurde er von der macedon〈ischen〉 Partei zum Tode verurtheilt u. entkam nach Aegina, wo er 322 auf Befehl Antipater's hingerichtet wurde. Von 77 Reden hielten die Alten 52 für echt ${ }^{160}$. Wir haben nur Fragmente, darunter 3 grössere. 1847

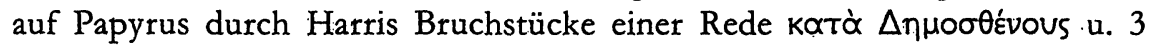

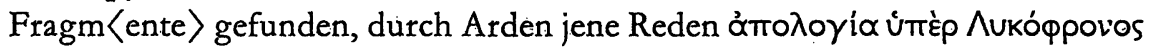

und der Kraft des Demosthenes. Der Ausdruck ist rein attisch, obgleich nicht ganz frei von Manier. Die Behandlung des Stoffes ist mit vielem Scharfsinn durchgeführt, und wenn auch zuweilen nicht sorgfältig genug, doch namentlich in Bezug auf Darlegung des Gegenstandes und Beweisführung sehr nachahmungswürdig. Vor Allem aber charakterisirt die Reden des Hyperides eine unnachahmliche Eleganz und Grazie."

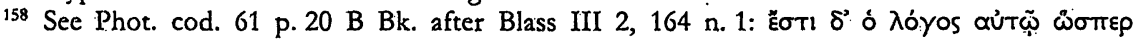

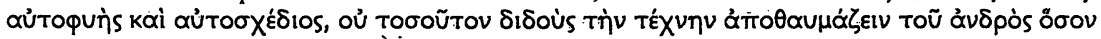
Tท่v фú

15") Cic. Or. 110.

${ }^{160}$ Quint. 10. 1. 77.

161 For the classical treatment of the Asian and Attic style see U. v. Wilamowitz-Moellendorff, "Asianismus und Atticismus", Kleine Scbriften III, edited by Friedrich Zucker, Berlin, 1969, 223-273, possibly composed with Nietzsche's Apollonine and Dionysiac in mind.

162 Also this paragraph is only a short note belonging to the more detailed treatment of Aeschines (IX). It derives from Westermann I, 117. Compare especially: "Dem entspricht wenigstens seine Fertigkeit im Extemporiren und der Charakter seiner Rede überhaupt, welche frei von aller Manier der reine Erguss eines übersprudelnden Genies ist. Hervorstechende Eigenschaft seiner Beredtsamkeit ist Kraft, Glanz und Fülle. Eindringlich und aufregend bei aller Leichtigkeit und bei aller Anmuth doch heftig und stụ̈rmisch, zeigt er mehr Fleisch als Muskel."

163 Dion. Hal. Dein. 1 (297. 1-14 (629 f.) U-R).

164. Hermogenes 1. 7,299. This is only a short note belonging to the more detailed treatment of Dinarchus (X). See p. 381. The material is clearly from Westermann 1, 157-159. Sec particularly $157 \mathrm{f:}$ " "Dinarchus ist nicht originell und hat eigentlich gat keinen besonderen rednerischen Charakter; er ahmt bald Lysias, bald Hyperides, namentlich aber Demosthenes nach". The reference to his tpaxútns is from Westermann I, 15.9 n. 19.

165 In contrast to Westermann Nietzsche changed the order and numbering of Hyperides and Aeschines. Here Nietzsche has used another source, or added material' on his own..

160 [Plut.] 849 D. 


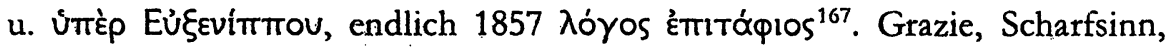
Prunk, poetische Färbung. Libanius giebt ihn für den Verf〈asser $\rangle$ von der

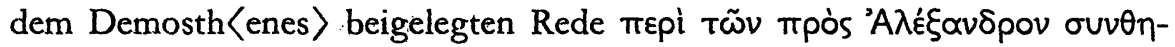
$\kappa \tilde{\omega} \nu^{168}$. Aeschines (IX) ${ }^{169}$ geb. $391^{170}$, niede〈rer $\rangle$ Geburt, Vorfechter im

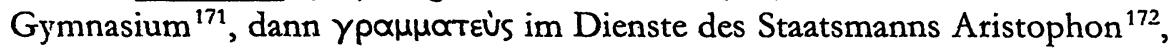
dann Schauspieler ${ }^{173}$, trat 356 gegen Philipp auf. Grosser Rathgeber. 347 wird er mit Demosth〈enes〉 zu Philipp geschickt, verräth sein Vaterland u. lässt sich bestechen. Jetzt wird er Todfeind des Demosth〈enes〉. Er ist jetzt Haupt der maced〈onischen〉 Partei. Katà TiứpXou gegen die Anklage auf Hoch-

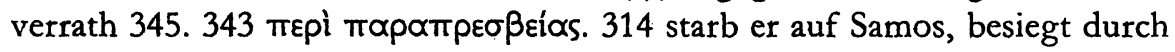

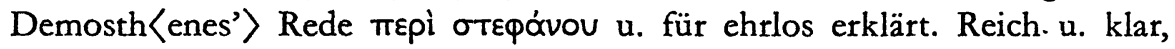
gewandt, wortreich, falsches Pathos ${ }^{174}$. Die berühmteste Rede Katஷ்

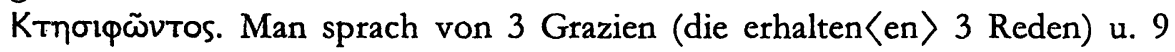
Musen des Aeschines $(9 \text { verlorene Briefe. })^{175}$.

Dinarchus $(\mathrm{X})^{176}$ geb. zu Corinth 361 , lebte zu Athen u. schrieb Reden für andere, bes〈onders〉 für die macedon〈ische〉 Partei. Sehr thätig als Werkzeug Antipater's u. während der Herrschaft des Demetrius Phalereus. 307 nach dessen Sturz verbannt. Durch Vermittl<ung $>$ Theophrast's erhielt er nach 15 Jahren die Erlaubnis der Rückkehr ${ }^{177}$ : Auf Befehl Polysperchon's wurde er 70 Jahre alt getödtet ${ }^{178}$. 160 Reden gab es ${ }^{179}$, nur $64^{180}$ oder $60^{181}$

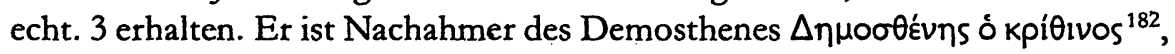
ordearius rhetor ${ }^{183}$, der "gerstene $\mathrm{D}\langle$ emosthenes〉" genannt. Demetrios ó

\footnotetext{
16: Papyri 1233-1236 Pack $^{2}$.

${ }^{168}$ Liban. VIII, 629 Foerster.

169 Here again Nietzsche is not directly based on Westermann.

176. Only some years later Blass III 2, 130 established the date of birth 390 or 389 . Westermann I, 113 has 389.

171 [Plut.] $840 \mathrm{~A}$.

172 See Dem. 19. 200; 237; 249 after Schäfer I² 254 n. 1.

${ }_{173}$ Demosthenes ridicules him as a tritagonistes: see Dem. 18. 261.

174 Dion. Hal. de imit. 5. 5; Quint. 12. 10. 23.

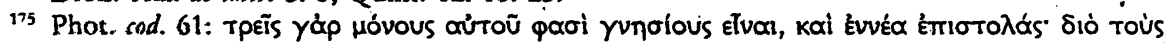

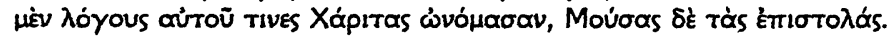

${ }_{176}$ No immediate source for Dinarchus is known to us.

17 Dion. Hal. Dein. 2 (300. 1-15 (633f.) U-R) after Westermann 1, 157-159.

1:8 This account derives from confusion with a Corinthian of the same name whom Antipater made commander in the Peloponnesus (Plut. Phoc. 33. 3-4, 757 A). The same crror can be found earlier in G. F.' Groddeck, Initia bistorica Graecarum lifterarum 1, Vilnas, 1821, 196.

${ }^{179}$ This account is based on Demetrius of Magnesia frag. p 52 Scheuerl., cited by Dion Hal. Dein. 1 (299. 2 (632) U-R). Dionysius later rejects it: see Dein. 2 (299. 13 (633) U-R).

[Plut.] $850 \mathrm{~F}$ und Phot. cod. 267.

56 Dionysius attributes to Dinarchus 60 genuine and 27 spurious speeches.

162 Because of his prolixity Hermogenes $I d .2 .11$ chatracterizes him in these terms:

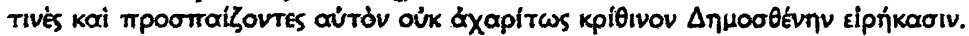

ies In superscript after Westermann 1, 159 n. 17.
} 
$\Phi \alpha \lambda \eta p \varepsilon$ U $^{18+}$ aus geringem Geschlecht, Zögling des Theophrast, durch glücklichste Naturanlagen zum mächtigen. Staatsmann werdend. Beginnt die polit〈ische〉 Laufbahn 325 zur Zeit der harpalischen Händel ${ }^{185}$. Nach Phocions Tode verwaltet er 10 Jahre Athen 317-307, unter Kassanders Regierung, zuerst zu allgem〈einer $\rangle$ Zufriedenheit ${ }^{186}, 360$ Statuen ihm errichtet ${ }^{187}$, dann bildet sich, bei seinem Übermuth u. seiner Ausschweifung, eine Partei Missvergnügter. Er entwich nach Theben, von da nach Aegypten: dort fand er gelehrte Musse, er lebt in vertrautem Umgange mit Ptolemaeus Soter. Ihm verdächtigt stirbt er in Oberaegypten 283. Sehr fruchtbarer Autor, 〈Diog.〉

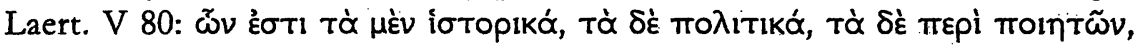

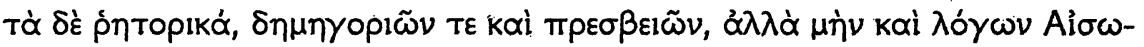

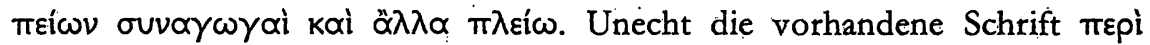

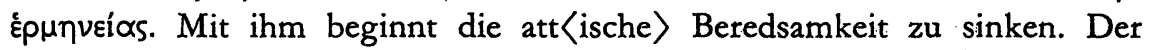
Charakter der Rede war sanft, weichlich, einschmeichelnd ${ }^{188}$, sein Ausdruck elegant, blühénd, ohne richtiges Maass. Der letzte att〈ische〉 Redner. Höchstens noch der Demosth〈enes〉 Schüler Cineas zu nennen, der mit Pyrrhus 280 nach Italien ging ${ }^{189}$.

Die rhodische Schule ${ }^{190}$, von Aeschines gegründet, der von Demosth〈enes〉 überwunden nach Asien ging u. zu Rhodus eine Schule stiftete ${ }^{191}$. Die eigentl〈iche〉 asianische von Hegesias aus Magnesia. Die thodische war die Vermittlerin der attischen und der asianischen, letztere viel stärker u. kräftiger, obgleich viel verwerflicher ${ }^{192}$. Cic. Brut. 13. 95 unterscheidet in der asian〈ischen〉 zwei Richtungen: genera autem Asiaticae dictionis duo sunt: unum sententiosum et argutum, sententiis non tam gravibus ${ }^{193}$ quam concinnis et venustis - aliud autem genus est non tam sententiis frequentatum quam verbis volucre et ${ }^{194}$ incitatum, quali est nunc Asia tota, nec flumine solum orationis sed etiam exornato et faceto genere verborum 125 . 1.) die

184 This part is again dependent on Westermann I, 159-161.

185 Diog. Laert. 5. 75.

186 See Diog. Laert. 5. 77. Cic. de leg. 3. 14.

187 This additional note " 360 Statuen ihm errichtet" is written in superscript. It comes from Westermann I, 161 n. 6. Ancient sources: Nepos Milt. 6 and Diog. Laert. 5. 75. GBP 184 read "360 Statuen von ihm errichtet" without indicating an addition to the text. "They fully misunderstand the matter.

188 Cic. Brut. 38. Orat. 92 after Westermann I, 160.

189 Plut. Pyrrbus 14. 1 after Westermann I, 161 n. 21.

190 This section is modelled on Westermann I, 164-166 and 175-180.

191 [Plut.] $840 \mathrm{D}$.

192 For a general characterisation see E. Norden, Die antike Kunstprosa 1, Stuttgart, 51958, $132 \mathrm{f}$.

193 Nietzsche carelessly copies Cicero's words from Westermann I, 176 n. 2 and omits “et severis".

194 An error for "atque".

${ }^{105}$ Cic. Brut. 95. 325. 
sententische Manier, in Verschlingung anmuthiger Gedanken, 2.) die verbo$\mathrm{se}^{196}$, immer strömend, mit zierlichen u. blumigen Worten ${ }^{197}$. Zu [sic] ersteren Hierocles u. Menecles aus Alabanda ${ }^{198}$, der zweiten Aeschylus aus Cnidus und Aeschines aus Milet ${ }^{199}$. Unter den rhodischen Rednern Apollonius, Molon genannt, aus Alabanda u. Hermagoras aus Temnos, berühmt noch mehr als Techniker. Merkwürdig, wie nun Athen von Asien wieder empfängt: es bildet sich in Athen eine asianische Beredsamkeit: Menedemus, Gastfreund des Antonius ${ }^{200}$, Demetrius aus Syrien, den Cicero hörte ${ }^{201}$, usw. Cicero selbst ist gebildet einmal durch Philosophen (Archias ${ }^{202}$, Phaedrus ${ }^{203}$, Diodotus ${ }^{204}$, Posidonius ${ }^{205}$, Philo ${ }^{206}$, Antiochus ${ }^{207}$ ) dann durch Redner: Xenocles ${ }^{208}$, Dionysius $^{209}$, Menippus ${ }^{210}$, Apollonius ${ }^{211}$. Té̉os [?] dabei: Wandlung des römischen Geistes durch griech〈ische〉 Cultur.|

Das Ereigniss [sic], das Rom der griech〈ischen〉 Beredsamkeit öffnete, war die Gesandtschaft der Athener 155 v. $\mathrm{C}\langle\text { hr. }\rangle^{212}$, um die Ermässigung einer wegen der Zerstörung von Oropos aufgelegten Geldstrafe zu erwirken, der Academ〈iker〉 Carneades, der Stoiker Diogenes, der Peripat〈etiker〉 Critolaus $^{213}$. Die Wirkung war so gross, dass Cato die Entfernung ${ }^{214}$ der

196 I. e. "die verbose Manier".

197 For the two-fold distinction compare the words of Westermann 1, 176: "die eine, die sentenziöse, gefiel sich in künstlicher Verschlingung mehr anmuthiger als inhaltsschwerer Gedankenreihen, die andere, die verbose, in unerschöpflichem Ausströmen eines in stattliche Zierrath eingedämmten Wortschwalles."

${ }^{198} \mathrm{Cic}$. Brut. 325. The brothers had been active since about 140 B.C. Cicero ibid. dates their active period to his own youth. See in general G. V. Sumner, "The orators in Cicero Brutus: Prosography and chronology", Pboenix, Supp. 11, Toronto, 1973.

.99 Cic. Brut. 325. Cicero heard Aeschylus 78 B. C. in Asia Minor; Cic. Brut. 316. Aeschines was of the same age as Cicero; Brut. 325.

2w Cic. de or. 1. 19. 85.

2in Cic. Brut. 315.

202 Cic. Arcb. 1.

203 Cic. Epist. 13. 1. 2; Fin. 1. 5. 16; 5. 1. 3; Leg. 1. 20.53.

as Inter alia Cic. Brut. 309; Acad. 2. 63. 115; Tusc. 5. 39. 113.

205 Cicero heard him in Rhodes; Plut. Cic. 4. 5, 862 F; Cic. Tuse. 2. 61; see also Fin. 1. 6; Nat. Deor. 1. 6.

306 Cic. Brut. 306.

an Cicero heard him in $79 / 78$ at Athens; Cic. Brut. 315. Cicero speaks of him elscwhere with respect: Acad. 1. 4. 13.

2008 Cic. Brut. 315.

2ff Diunysios of Magnesia is meant; sec Cic. Brut. 316.

210 Cic. Brut. 315.

211 In 81 Apollonius came to Rome, in 78 Cicero visited him in Rhodes (Brut. 312). Cicero praises the moderating influence Apollonius had on him (Brut. 316. 325).

212 For the report about the Athenian mission see Westermann I, $167 £$.

${ }^{213}$ For the delegatiun of the Greek philosophers see Gellius 6 (7). 14. 8.

214 GBP 186 read: "Der Academiker Corneades [sic], der Richter Diogenes, der Peripatetiker A ristolaus. Die W'irkung w'ar so gross, dass Cato die Betreuung der Gesandten antrug." 
Gesandten antrug ${ }^{215} .161$ hatte man senatus consultum de philosophis et rhetoribus Latinis gemacht, uti Romae non [sic] essent ${ }^{216}$. Danach aber, 6 Jahre später, sagte der Senat Aelian V〈aria〉 H〈istoria〉 III 17: ह̌meququv

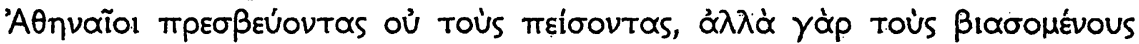

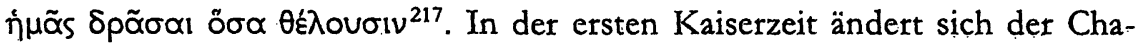
rakter der Beredsamkeit nicht. Die Schulen in Athen verloren etwas, der Zug der röm〈ischen〉 Jugend ging nach Massilia oder Asien, wo Tarsus von Rednern wimmelte. Die Redekunst ist die Liebhaberei der studierenden Jugend. Schule zu Mytilene auf Lesbos, Timocrates - Lesbonax - Potamon, Lehrer u. Freund des Tiberius ${ }^{218}$. In Asien ist Theodorus aus Gadara Stifter

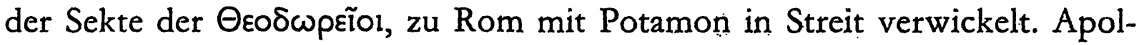
lodorus aus Pergamus ${ }^{219}$, Stifter der pergamen〈ischen〉 Sekte der 'Aто $\lambda$ $\lambda \circ \delta \omega p \varepsilon i ̃ o l . ~ B e r u ̈ h m t e r$ als alle Dio, Chrysostomus ${ }^{220}$ zubenannt, aus Prusa in Bithynien. In der Heimat verkannt geht er nach Rom, ist dort Domitian verdächtig (Gell. N. A. XV $11^{221}$, philosophi $\langle\ldots\rangle$ Domitiano imperante senatus consulto ejecti atque urbe et Italia interdicti sunt), entweicht aus Rom u. beginnt angebl〈ich〉. auf Rath des delph〈ischen $\rangle$ Orakels ${ }^{222}$ im Bettlerkleide $^{223}$ eine Wanderung durch Thracien, Illyrien, Scythien u. das Land der Geten, nichts als Platons Phaedon u. Demosth〈enes' $\rangle$ Rede de falsa legatione in der Tasche ${ }^{224}$. Mit Ehren überhäuft kehrt er nach Prusa zurück ${ }^{225}$. Nach Domitians Ermordung 96 c. stimmt er die Grenzarmeen zu Gunsten seines Freundes ${ }^{226}$ Coccejus Nerva (daher der Beiname [?] Cocceianus ${ }^{227}$ ) u. ging nach Rom, sehr geehrt von dort nach Prusa, das ihm bald durch Kleinstädterei verleidet wird ${ }^{228}$. In Rom stirbt er, in hoher Achtung bei Trajan 117 pC. Es

215 Plut. Cato 22.

216 Gell. 15. 11. 1. Latinis is regularly deleted by modern editors after Pighius comparing Suet. de rbet. 1. Obviously Nietzsche had copied it from Westermann 1, 168 n. 6. Further, he replaces Gellius' ne with non; see Kühner-Stegmann, Ausfübrliche Grammatik der lateinischen Sprache II, Leverkusen, ${ }^{3} 1955, \$ 1842 \mathrm{ab}$. It is correct in Westermann.

217 48. 7-9 Dilts. This citation is taken from Westermann I, $168 \mathrm{n} .4$.

${ }^{218}$ Nietzsche copies an error from Westermann I, 186. In fact not Potamon, but Theodorus of Gadara was Tiberius' teacher (Suet. Tib. 57): see F. Susemihl, Geschichte der griechischen Literatur in der Alexandrinerzeit II, Leipzig, 1892, 514 n. 228.

219 Pergamon is meant. Westermann I, 186 too has "Pergamus".

220 See Westermann I, 189- 191.

221 15. 11. 3-4. Citation from Westermann I, 190 n. 5.

222 Dio. Chrys. 13. $9 \mathrm{f}$.

223 Dio. Chrys. $12.85 ; 13.10$.

224 See Philostrat. Soph. 448. The sentence is from Westermann I, 190 n. 7.

225 A direct citation from Westermann I, 189.

220 Philostrat. Soph. 488.

227 Plin. Ep. 10. 85; see Westermann 1, 191 n. 9. The reading is uncertain.

228 See the almost literal rendering from Westermann I, 189: "allein dèr dort herrșchende kleinstädtische Geist [...] verleidete ihm den Aufenthalt daselbst". 
sind 80 Reden erhalten: wenig davon gehört der ersten Periode an; seine Form ist bes〈onders〉 nach Hyperides $u$. Aeschines gebildet, die er als Muster selbst Demosth〈enes〉 u. Lysias vorzog ${ }^{229}$. Dionys〈ius〉 v. Halicarn〈ass〉, der bedeutendste rhetor $\langle$ ische $\rangle$ Kunstrichter ${ }^{230}$, in den Schulen Asiens gebildet, kam 25 Jahre alt 29 a. Ch. nach Rom ${ }^{231}$, hauptsächlich um römische

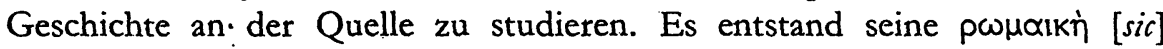

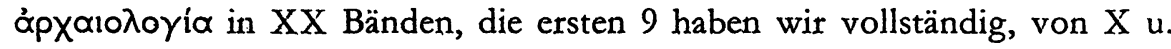
$\mathrm{Xl}$ nur den grössten Theil und Auszüge der übrigen. Eine Rhetorik unter seinem Namen ist ein Cento aus 4 Hauptpartien, theilweise von Dionys $\langle\text { ius }\rangle^{232}$. Sehr wichtige Schriften verloren, aber erhalten $\pi \varepsilon p i ̀ ~ \sigma u \nu \theta \varepsilon ́ \sigma \varepsilon \omega s$

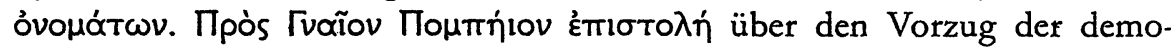
sth〈enischen〉Schreibart vor der platonischen. 'Emı

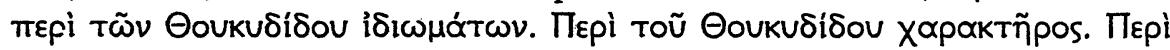

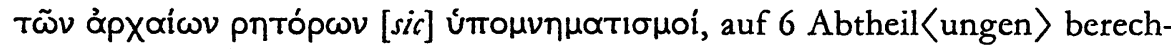
net, von denen aber nur die erste Hälfte, 1. Lysias. 2. Isocrates. 3. Isaeus, u.

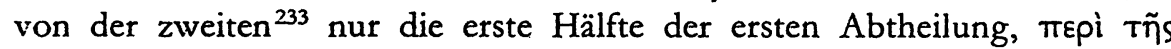

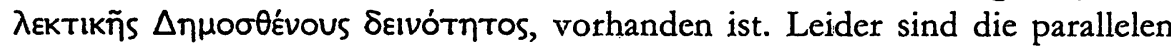

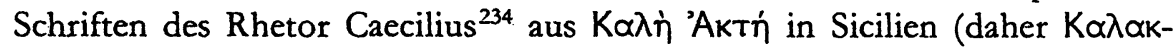
Tivo5) verloren. Schriften genannt bei Suidas ${ }^{235}$. Echtheitsentscheidung über die älteren Redner. - Eine neue Epoche beginnt mit Hadrian ${ }^{236}$ : Athen blüht auf. Mark Aurel gründet zwei öffentl〈iche〉Schulen ${ }^{237}$, eine philosophische u. eine rhetorische, die erstere mit 4 Kathedern ${ }^{238}$ (nach den 4 Hauptschulen, von jeder 2 Professoren), die leztere [sic] in 2 Apóvol, in das sophistische u. das politische Fach. Die Profess〈oren〉 erhielten jährlich 10000 Drachmen. Später stieg für jedes Katheder die Zahl der Lehrer bis auf 6. Kraft kaiserl〈ichen〉Willens wird der Name Sophist wieder zu Ehren gebracht ${ }^{239}$. Ausserordentl<icher〉 Wetteifer bei grosser Entartung, hohe Koketterie der Deklamierenden. Lucian entwirft ein starkes Bild von dieser Entartung.

225y See W'estermann I, 191 n. 20.

230. See Westermann I, 192f. and in general S. F. Bonner, The Literary Treatises of Dionysius of Halicarnassus: $A$ Study in the Development of Critical Metbod, Amstcrdam, 1969.

231 Dion. Hal. Ant. Rom. 1. 7.

232 See Westermann 1, 194 n. 4.

23) The contents of the second half: 4. Demosthenes, 5. Hyperides, 6. Aeschines.

24. See Westermann 1, 193 and 197 nn. 16 and 17 and Caecilii Calactini Fragmenta, collegit Ernestus Ofenloch, Jeipzig, 1907, repr. Stuttgart, 1967.

23. K 1165 Adler.

25n For the following see Westermann 1, 198-202. Note the almost literal citation from Westermann L, 198 \&.

23: For Marcus Aurelius' founding of the University of Athens see J.W. H. Whalden, The Iniversities of Ancient Greect, New York, 1909, 90-94 and the sources there cited.

2365 Luc. I:un. 3.

29. See G. W'. Bowersock, Greek Sophists in tbe Roman Empire, Oxford. 1969. 
Berühmt in Athen vor Allen Herodes Atticus, in hoher Gunst bei den Antoninen. Grosser Improvisator. Schriften verloren ${ }^{240}$. In Asien berühmt Aclius Aristides $^{241}$ aus Adriani in Mysien geb. 129. Noch 55 Reden u. Abhandl〈ungen〉 erhalten, 2 rhetor〈ische〉 Schriften von geringem Werthe $^{242}$. Lucian ${ }^{243}$ aus Samosata 130-200 n. Chr., früher selbst Sophist u. Rhetor $^{244} \mathrm{u}$. wendet sich 40 Jahre alt zur Philosophie, ankämpfend gegen alles Sophistenthum ${ }^{245}$. Ausserordentlicher Techniker, also Kunstschriftsteller, ist Hermogenes $^{2+6}$ aus Tarsus, ingenium praecox, im $15 t\langle e n\rangle J\langle a h r\rangle$ öffentl〈icher〉 Lehrcr, im 17t〈en〉 Schriftsteller, im 25〈ten〉 unheilbare Gei-

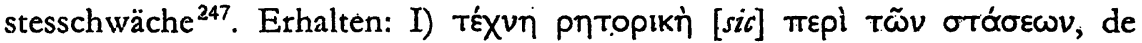

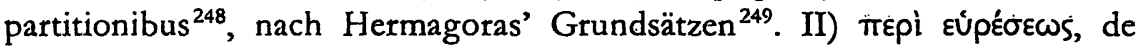
invent〈ione $\rangle^{250}$, in 4 Büchern. III) $\pi \varepsilon p i$ i $\delta \varepsilon \tilde{\omega} \nu$, de formis oratoriis ${ }^{251}$, in 2

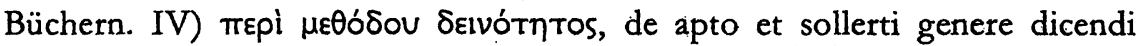
methodus $^{252}$. V) $\pi \rho \circ \gamma u \mu \nu \alpha ́ \sigma \mu \alpha T \alpha$, praeexercitamenta ${ }^{253}$.

Die drei Philostrati ${ }^{254}$ : I) Flavius Philostratus, Sohn des Verus aus Hierapolis, lebt noch unter Severus. II) des ersteren Sohn, erst Lehrer in Athen, dann in Rom, Biograph des Apollonius $v\langle$ on〉 Tyana auf Verlangen der

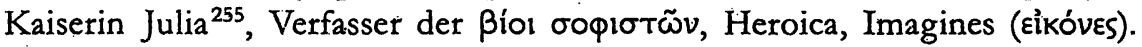
III) des zweiten Schwestersohn, in Gunst bei Caracalla, starb unter Galienus

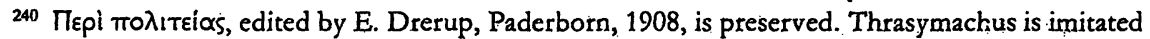
so perfectly that some wanted to date this speech to the fifth century. See K. Münscher, $R E$ 8 (1912); 921-954; 1308-1310.

241 See Westermann I, 209-212. But from here on his excerpts get so short that there are hardly any parallels to be found.

${ }^{242}$ Nietzsche follows Westermann I, 210. Modern opinion holds that both works were attributed to Aristeides relatively late: See Christ-Schmid-Stählin, Geschicbte der griecbiscben Litteratur II 2, München, ${ }^{6} 1924,699$ n. 8.

243 See Westermann I, 213.

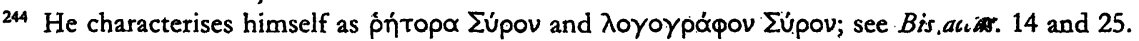

${ }^{245} \mathrm{He}$ even compares rhetoric to a rouge painted hetaira; Bis accus. 31.

246 See Westermann I, 217-220.

${ }^{247}$ Proleg. Hermog. de Stat. 2. 222. 12 Walz after Westermann I, 217.

248 In superscript after Westermann I, 219 n. 4.

249 See Westermann I, 218.

250 In superscript after Westermann I, 219 n. 5.

251 In superscript after Westermann I, 219 n. 6.

${ }^{252}$ In subscript after Westermann I, 219 n. 7.

253 See Westermann I, 220 n. 8. GBP 190 read: "Ausserordentlicher Darsteller also Kunstschriftsteller ist Hermogenes aus Tarsus, ingenium praecox, im 15. Lebensjahr öffentlicher Lehrer, im 17. Jahre Schriftsteller, im 25. unfehlbarer Geist in der Sprache. Erhalten: (I) TéXvn

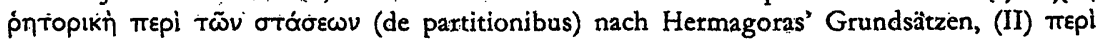

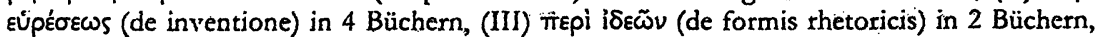

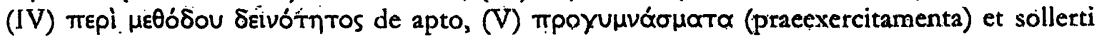
generi methodas."

254 See Westermann I, 223-226.

255 Vit. Ap. 1. 3; the empress Julia Domna is meant. 
$[\text { sic }]^{256}$ 264. Feiner Kunstkritiker. Dionysius Cassius Longinus 213-273, berühmt $\pi \varepsilon p i$ ü $\psi o u{ }^{257}$. Verfasser von vorhandenen $\pi p \circ \gamma \cup \mu v \alpha \dot{\sigma} \sigma \mu \alpha \tau \alpha$ sind Aphthonius aus Antiochien u. Aelius Theon aus Alexandria ${ }^{258}$. Himerius ${ }^{259}$ aus Prusias in Bithynien c. 315-386, berühmter Lehrer in Athen. Von 71 Reden ${ }^{260}$, die Photius nannte, besitzen wir noch 36 von diesem gemachte

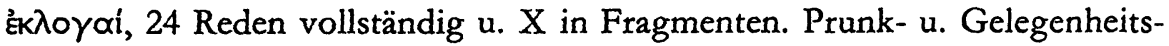
reden. Sein Schüler ist der Kaiser Julianus 331-363. In Byzanz blüht bis auf Theodosius von Constantin〈opel〉u. Julianus, Themistius, wir besitzen 34 Reden $^{261}$. In Asien blüht Libanius ${ }^{262}$ aus Antiochien, ausserordent〈lich〉 fruchtbar. Erhalten $66^{263}$ Reden, $50^{264}$ Deklamationen. Dann Musterstücke zu rhetor〈ischen〉 Vorübungen ${ }^{265}$. Dann die bekannten Inhaltsanzeigen der Reden des Demosth〈enes ${ }^{266}$ u. Biographie des Demosth $\langle\text { enes }\rangle^{267}$; das letzte grosse Talent ${ }^{268}$.

\section{III.}

\section{What bave we learned?}

Certainly nothing we did not know about the Greek orators. Otto Crusius rightly remarked 269: "Der verkürzte Abriss der Geschichte der Beredsamkeit hinter der Darstellung der Rhetorik hat neben dieser ausführlichen Form [i.e. the 1874 notes] keine selbständige Bedeutung." But we have learned that Germanists innocent of Greek and Latin ought not to edit works of Nietzsche that require Greek and Latin. We have also learned something

256 Nietzsche's error for "Gallienus".

${ }^{257}$ The author today is regularly called Ps.-Longinus. Nietzsche does not know Aemil. Winkler, De Longini qui fertur libello mepi üyous, Diss. Halle, 1870, who proved the whole spurious on linguistic grounds. Nietzsche uncritically follows Westermann I, 229-232, who (230 and 232 n. 9) explicitly defends the work as genuine.

25s See Westermann I, 230 and $233 \mathrm{f}$.

${ }^{254}$ For Himerius and Julian see Westermann I, 239-242.

20*) Here Nietzsche errs (after Westermann 1, 240), Photius still knew 73, not 71; see Phot. bibl. $107-109$ und 353-377 Bekker.

202 So Westermann I, 243 and $244 \mathrm{n}$. 9 . Only. 33 speeches are preserved; he adds the twelfth speech, which is judged as a modern forge in the more recent research; see Westermann I, $328-330$, Beilage XIV.

242 See Westermann 1, 244-249. For the following text sce Westermann 1, 246.

263 See W'estermann 1, 248 n. 28.

$3 / 4$ Westermann I, 248 n. 29.

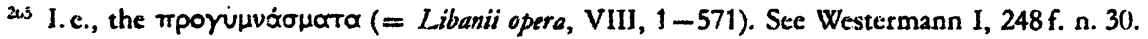

2se Libanii opera, VIII, ed. R. Foerstet, Leipzig, 1915, 573-681.

26: Libanii opera, VIII, 600-607.

2ye Sce G. R. Sievers, Das Leben des Libanius, Berlin, 1868, rcpr. Amsterdam, 1969.

3s" Op. cit. (supra n. 2), 331. 
about the man Nietzsche and about his ścholarly attention to Greek antiquity. The Abriss is written by a man about a subject that bores him. The work is Pflicht rather than Neigung. He needs a minimum of fact to support the theory of the Darstellung der Antiken Rbetorik. The two students might ask questions. It was a time of disappointment and even humiliation. Presumably Nietzsche had chosen the subject, because students ought to read the orators and would not on their own. Then unexpectedly all the students who needed the orators boycotted his course. What possible reason was there to spend time and do well?

There are two revealing facts that emerge from the study of the essay: Nietzsche's grasp of Greek and Latin certainly does not equal that of his younger contemporary and fellow Pforte graduate, Ulrich von WilamowitzMoellendorff. One need only recall the solecism $\dot{\varepsilon} v \tau \tilde{n}$ бopı $\sigma \tau \tilde{\varphi}^{270}$ and the indifference to particles ${ }^{271}$. He was not a master of Greek and Latin ${ }^{272}$ grammar. As far as the composition of the essay goes, we have shown that it is almost entirely derivative from two preedecessors, Fr. Blass and A. Westermann. Both these books were in his personal library. Neither is annotated ${ }^{273}$. He had little independent knowledge of the subject as his omission of Isocrates' most famous oration and his naive attribution of $\dot{d} e$ sublimitate to Longinus prove. Nor did it trouble him to lecture to students about authors whom he had never read presenting as his own conclusions what he had merely excerpted from contemporary handbooks, assured that his students were too lazy to unmask him.

Albert Henrichs has recently demonstrated that, while Nietzsche wrote in 1872 perhaps the most influential damnatio of Euripides, in fact he does not reveal personal knowledge of any Euripidean tragedy other than Bacchae, which he knew well. His entire criticism of Euripides was derived ffom A. W. Schlegel ${ }^{274}$. "The fundamentals of Nietzische's case against Euripides are demonstrably derived from Schlegel's Vienna lectures." 275 In spite of Wilamowitz' telling refutation of the philological and historical basis of Geburt,

270 See supra n. 8.

271 See supra n. 127.

272 See supra n: 216.

273 Max Oehler, Nietzsches Bibliothek. Vierzebnte Jabresgabe der Gesellschaft der Freunde des NietzscbeArchivs Weimar, Weimar, 1942, 9, 16. In November 1872 he borrowed the University Library's copy of Blass I (Oehler, 51).

274 Albert Henrichs, "The-last of the detractors: Friedrich Nietzsche's condemnation of Euripides", GRBS 27 (1986), 369-397, esp. 37.6-385 ("Euripides at Second Hand: Nietzsche's Use of A. W. Schlegel”). For Schlegel's critique of Euripides see Ernst Behler, “A. W. Schlegel and the nineteenth-century damnatio of Euripides", $G R B S 27$ (1986), 335-367.

275 ibid., 384. 
Nietzsche continued with oratory, as he had earlier with Euripidean tragedy, to base his case on secondary literature rather than original sources. Probably the most important contribution of the new text is that it confirms Henrichs' discovery for Geburt and further illustrates the welcome truth that by November 1872 traditional historical philological scholarship bored Friedrich Nietzsche. Incipit tragoedia ${ }^{276}$.

"s See Curt Paul Janz, Fritdricls Nietzscbe. Biograpbie 1, Munich/Vienna, 1978, 494. 